\title{
A Class of Integrable Flows on the Space of Symmetric Matrices
}

\author{
Anthony M. Bloch* \\ Department of Mathematics \\ University of Michigan \\ Ann Arbor MI 48109, USA \\ abloch@math.lsa.umich.edu \\ Vasile Brînzănescu ${ }^{\dagger}$ \\ "Simion Stoilow" Institute of Mathematics of the Romanian Academy \\ P.O.Box 1-764, 014700 Bucharest, Romania and \\ Department of Mathematics and Informatics \\ University of Piteşti \\ 110040 Piteşti, Romania \\ Vasile.Brinzanescu@imar.ro \\ Arieh Iserles \\ Department of Applied Mathematics and Theoretical Physics \\ University of Cambridge \\ Wilberforce Road \\ Cambridge CB3 0WA, UK \\ A.Iserles@damtp.cam.ac.uk \\ Jerrold E. Marsden $\ddagger$ \\ Control and Dynamical Systems 107-81 \\ California Institute of Technology \\ Pasadena, CA 91125, USA \\ marsden@cds.caltech.edu \\ Tudor S. Ratiu ${ }^{\S}$ \\ Section de Mathématiques and Bernoulli Center \\ Ecole Polytechnique Fédérale de Lausanne \\ CH-1015 Lausanne, Switzerland \\ Tudor.Ratiu@epfl.ch
}

This version: March 22, 2009

\footnotetext{
* Research partially supported by NSF grantsCMS-0408542 and DMS-604307.

$\dagger$ Research partially supported by the Swiss SCOPES grant IB7320-110721/1, 2005-2008, and MEdC Contract 2-CEx 06-11$22 / 25.07 .2006$

${ }^{\ddagger}$ Research partially supported by the California Institute of Technology and NSF-ITR Grant ACI-0204932.

${ }^{\S}$ Research partially supported by the Swiss NSF and the Swiss SCOPES grant IB7320-110721/1.
} 


\begin{abstract}
For a given skew symmetric real $n \times n$ matrix $N$, the bracket $[X, Y]_{N}=X N Y-Y N X$ defines a Lie algebra structure on the $\operatorname{space} \operatorname{Sym}(n, N)$ of symmetric $n \times n$ real matrices and hence a corresponding Lie-Poisson structure. The purpose of this paper is to investigate the geometry, integrability, and linearizability of the Hamiltonian system $\dot{X}=\left[X^{2}, N\right]$, or equivalently in Lax form, the equation $\dot{X}=[X, X N+N X]$ on this space along with a detailed study of the Poisson geometry itself. If $N$ has distinct eigenvalues, it is proved that this system is integrable on a generic symplectic leaf of the Lie-Poisson structure of $\operatorname{Sym}(n, N)$. This is established by finding another compatible Poisson structure.

If $N$ is invertible, several remarkable identifications can be implemented. First, $(\operatorname{Sym}(n, N),[\cdot, \cdot])$ is Lie algebra isomorphic with the symplectic Lie algebra $\mathfrak{s p}\left(n, N^{-1}\right)$ associated to the symplectic form on $\mathbb{R}^{n}$ given by $N^{-1}$. In this case, the system is the reduction of the geodesic flow of the left invariant Frobenius metric on the underlying symplectic group $\operatorname{Sp}\left(n, N^{-1}\right)$. Second, the trace of the product of matrices defines a non-invariant non-degenerate inner product on $\operatorname{Sym}(n, N)$ which identifies it with its dual. Therefore $\operatorname{Sym}(n, N)$ carries a natural Lie-Poisson structure as well as a compatible "frozen bracket" structure. The Poisson diffeomorphism from $\operatorname{Sym}(n, N)$ to $\mathfrak{s p}\left(n, N^{-1}\right)$ maps our system to a Mischenko-Fomenko system, thereby providing another proof of its integrability if $N$ is invertible with distinct eigenvalues. Third, there is a second ad-invariant inner product on $\operatorname{Sym}(n, N)$; using it to identify $\operatorname{Sym}(n, N)$ with itself and composing it with the dual of the Lie algebra isomorphism with $\mathfrak{s p}\left(n, N^{-1}\right)$, our system becomes a Mischenko-Fomenko system directly on $\operatorname{Sym}(n, N)$.

If $N$ is invertible and has distinct eigenvalues, it is shown that this geodesic flow on $\operatorname{Sym}(n, N)$ is linearized on the Prym subvariety of the Jacobian of the spectral curve associated to a Lax pair formulation with parameter of the system. If, on the other hand, $N$ has nullity one and distinct eigenvalues, in spite of the fact that the system is completely integrable, it is shown that the flow does not linearize on the Jacobian of the spectral curve.
\end{abstract}

\title{
Contents
}

1 Introduction

2 The Lie Algebra and the Euler-Poincaré Form

3 Poisson Structures

4 The Sectional Operator Equations and Relation to Mischenko-Fomenko Flows

5 Lax Pairs with Parameter

6 Involution

7 Independence

8 Linearization of the Flow

\section{Introduction}

The Problem and Discussion of the Results. Fix $N \in \mathfrak{s o}(n)$, the space of skew symmetric $n \times n$ matrices, also regarded as the Lie algebra of $\mathrm{SO}(n)$, the $n$-dimensional proper orthogonal group. This paper continues the analysis, begun by Bloch and Iserles in [5], of the following set of ordinary differential equations on $\operatorname{Sym}(n)$, the linear space of $n \times n$ symmetric matrices:

$$
\dot{X}=\left[X^{2}, N\right] .
$$

Here, $X \in \operatorname{Sym}(n), \dot{X}$ denotes the time derivative, and initial conditions are denoted $X(0)=X_{0} \in \operatorname{Sym}(n)$. It is easy to check that $\left[X^{2}, N\right] \in \operatorname{Sym}(n)$, so that if the initial condition is in $\operatorname{Sym}(n)$ then $X(t) \in \operatorname{Sym}(n)$ 
for all $t$. As will be seen shortly, this system is Hamiltonian and, despite its quadratic dependence on $X$, conservation of energy guarantees that solutions of (1.1) exist for all $t \in \mathbb{R}$.

Because of the obvious identity $\left[X^{2}, N\right]=[X, X N+N X]=X^{2} N-N X^{2}$, equation (1.1) may be rewritten in the Lax form

$$
\dot{X}=[X, X N+N X]
$$

again with initial conditions $X(0)=X_{0} \in \operatorname{Sym}(n){ }^{1}$

Define the $N$-bracket by $[X, Y]_{N}:=X N Y-Y N X$. It is easy to check that this makes $\operatorname{Sym}(n)$ into a Lie algebra and with this structure it will be denoted $\operatorname{Sym}(n, N)$. The structure of this Lie algebra is completely analyzed in the present paper. Using the trace inner product, identify $\operatorname{Sym}(n, N)$ with its dual and endow it with the associated Lie-Poisson structure. As will be done below, it is straightforward to show that the system (1.1) is Hamiltonian with respect to this Lie-Poisson structure with Hamiltonian equal the quadratic form defined by the Frobenius metric. Interestingly, the system is also Hamiltonian with respect to a compatible "frozen" Poisson structure; this provides a bi-Hamiltonian structure for equation (1.1). We study the Poisson geometry on $\operatorname{Sym}(n, N)$ for both Poisson structures and, in particular, determine the generic leaves and the Casimir functions of both Poisson structures relative to which the system (1.1) is bi-Hamiltonian. The Poisson geometry in the case $N$ is not invertible turns out to be particularly rich.

A key result of the paper is that if $N$ has distinct eigenvalues (one of which could be zero), this system is integrable on the generic symplectic leaf of $\operatorname{Sym}(n, N)$ (of either the Lie-Poisson or the frozen Lie-Poisson structures). The proof makes use of the Lax pair with parameter found in [5] to find a class of integrals that, as we show using the preceding bi-Hamiltonian structure together with a technique inspired by [22], are in involution. ${ }^{2}$ Related work on bi-Hamiltonian structures may be found in [17] and [6]. Independence is proved directly.

We show that if $N$ is invertible, the Lie algebra $\operatorname{Sym}(n, N)$ is isomorphic to the symplectic Lie algebra $\mathfrak{s p}\left(n, N^{-1}\right)$, where the symplectic form on $\mathbb{R}^{n}$ is given by $N^{-1}$. Thus, in this case, the system (1.1) is Lie-Poisson on (the dual of) $\mathfrak{s p}\left(n, N^{-1}\right)$, and so the system is the (Euler-Poincaré or Lie-Poisson) reduction of the geodesic flow on the underlying symplectic group, denoted by $\operatorname{Sp}\left(n, N^{-1}\right)$, relative to the Frobenius metric.

If $N$ is invertible there is a Poisson diffeomorphism from $\mathfrak{s p}\left(n, N^{-1}\right)$ to $\operatorname{Sym}(n, N)$, the inverse of which maps our system to a Mischenko-Fomenko system $(\text { see }[19 ; 20 ; 21])^{3}$, thereby providing another proof of integrability in the case that $N$ is invertible with distinct eigenvalues. In addition, by identifying the symmetric matrices with themselves by an an ad-invariant inner product if $N$ is invertible (as opposed to the standard identification by the trace of the product used before which is valid in general, even if $N$ is not invertible), our flow can be seen as a Mischenko-Fomenko flow on its dual. A byproduct of our work is thus the bi-Hamiltonian structure for the associated Mischenko-Fomenko system on $\mathfrak{s p}\left(n, N^{-1}\right)$. Bi-Hamiltonian structures for Mischenko-Fomenko systems were first discussed in [17], [6], and later in [22]. We also note that the sequence of integrals we produce by our Lax pair with parameter method on $\operatorname{Sym}(n, N)$ is not produced by shifting the arguments in Casimir functions. Relative to the Lie-Poisson structure on $\operatorname{Sym}(n, N)$, our method for analyzing this system appears to be fundamentally different from completely integrable systems either of rigid body or Toda type (on symmetric matrices) and none of the standard involution theorems (see e.g. [25]) seem to be applicable.

Since the system (1.1) is integrable and its integrals are polynomials, one would expect that this system may be algebraically completely integrable (as defined, for example, in [3]). It turns out that the situation is quite involved.

If $N$ is invertible and has all eigenvalues distinct, then the linearization criterion in [3] or [11] applies and the system is linearizable on the Jacobian of the associated spectral curve. In spite of this fact, we could not prove that the system is algebraically completely integrable. However, the spectral curve has an involution, and thus the system is in fact linearizable on a Prym variety.

\footnotetext{
${ }^{1}$ Integrable equations that bear a formal resemblance to equation (1.1); that is, to (1.2), in the context of free associative algebras are given in [18] and [24].

${ }^{2} \mathrm{~A}$ related result on bi-Hamiltonian structures for rigid body type equations with a parameter can be found in [7]. Note that the bi-Hamiltonian structure in the present paper is for the equations without parameter, which is more relevant for the present study.

${ }^{3}$ We thank A. Bolsinov for this observation and the referee for a related observation.
} 
If $N$ has odd size, distinct eigenvalues, and nullity one, we show by the concrete study of the case $n=5$ that the system (1.1) is not linearizable on the Jacobian of the spectral curve. On the other hand, it was already shown that the system is integrable, so this situation is an example of an integrable system all of whose integrals are polynomials but whose flow does not linearize on the Jacobian of the spectral curve.

The Structure of the Paper. In $\S 2$, the Lie algebra structure on the space of symmetric matrices induced by $N$ is introduced and in the case in which $N$ is invertible, the isomorphism with $\mathfrak{s p}\left(n, N^{-1}\right)$ is set up. In $\S 3$, two compatible Poisson structures are defined and the associated bi-Hamiltonian structure is analyzed, and the symplectic leaves and Casimir functions of both Poisson structures are determined. In $\S 4$ the system (1.1) is shown not to directly lie in this family. However, the dual of a Lie algebra isomorphism defines a Poisson isomorphism from $\mathfrak{s p}\left(n, N^{-1}\right)$ to $\operatorname{Sym}(n, N)$; its inverse maps (1.1) to a Mischenko-Fomenko system on $\mathfrak{s p}\left(n, N^{-1}\right)$ if $N$ has distinct eigenvalues. This fact provides a proof of complete integrability of (1.1) if $N$ is invertible with distinct eigenvalues. $\S 5$ returns to the system (1.1) on $\operatorname{Sym}(n, N)$, presents the Lax pair with parameter, and finds a new family of functions containing the right number of functionally independent integrals of motion; this set of functions is thus a candidate for the Liouville integrals. In $\S 6$ involutivity of these integrals is shown using the bi-Hamiltonian structure and $\S 7$ proves the independence of these functions provided that $N$ has distinct eigenvalues and is either invertible or has nullity one. Finally, $\S 8$ is devoted to the proofs of the linearization statements given above.

\section{The Lie Algebra and the Euler-Poincaré Form}

Regarding $N$ as a Poisson tensor on $\mathbb{R}^{n}$, the bracket of two functions $f, g$ is defined in the standard way as

$$
\{f, g\}_{N}=(\nabla f)^{T} N \nabla g .
$$

The Hamiltonian vector field associated with a function $h$ (with the convention that $\dot{f}(z)=X_{h}(z) \cdot \nabla f(z)=$ $\{f, h\}(z))$ is easily checked to be given by

$$
X_{h}(z)=N \nabla h(z) .
$$

Quadratic Functions. For each $X \in \operatorname{Sym}(n)$, define the quadratic Hamiltonian $Q_{X}$ by

$$
Q_{X}(z):=\frac{1}{2} z^{T} X z, \quad z \in \mathbb{R}^{n} .
$$

Let $\mathcal{Q}:=\left\{Q_{X} \mid X \in \operatorname{Sym}(n)\right\}$ be the vector space of all such functions. Note that the map $Q: X \in$ $\operatorname{Sym}(n) \mapsto Q_{X} \in \mathcal{Q}$ is an isomorphism. Using (2.2) it follows that the Hamiltonian vector field of $Q_{X}$ has the form

$$
X_{Q_{X}}(z)=N X z .
$$

The Poisson bracket of two such quadratic functions is easy to work out.

Lemma 2.1 For $X, Y \in \operatorname{Sym}(n)$, we have

$$
\left\{Q_{X}, Q_{Y}\right\}_{N}=Q_{[X, Y]_{N}},
$$

where, as earlier, $[X, Y]_{N}:=X N Y-Y N X \in \operatorname{Sym}(n)$. In addition, $\operatorname{Sym}(n)$ is a Lie algebra relative to the Lie bracket $[\cdot, \cdot]_{N}$ and with this structure will be denoted $\operatorname{Sym}(n, N)$. Therefore, $Q: X \in\left(\operatorname{Sym}(n, N),[\cdot, \cdot]_{N}\right) \mapsto$ $Q_{X} \in\left(\mathcal{Q},\{\cdot, \cdot\}_{N}\right)$ is a Lie algebra isomorphism.

Proof. Using (2.1), we have

$$
\begin{aligned}
\left\{Q_{X}, Q_{Y}\right\}_{N}(z) & =\left(\nabla Q_{X}\right)(z)^{T} N\left(\nabla Q_{Y}\right)(z)=(X z)^{T} N Y z=z^{T} X N Y z \\
& =\frac{1}{2} z^{T}(X N Y-Y N X) z=Q_{[X, Y]_{N}}(z) .
\end{aligned}
$$


Recall that the notation $Q_{V}$ is reserved only for symmetric matrices $V$. Since $X, Y \in \operatorname{Sym}(n, N)$ implies that $[X, Y]_{N}=X N Y-Y N X \in \operatorname{Sym}(n, N)$ we can write $Q_{[X, Y]_{N}}$ in the preceding equation.

The bracket $[\cdot, \cdot]_{N}$ on $\operatorname{Sym}(n, N)$ is clearly bilinear and antisymmetric. The Jacobi identity follows by a straightforward direct verification.

It is a general fact that Hamiltonian vector fields and Poisson brackets are related by

$$
\left[X_{f}, X_{g}\right]=-X_{\{f, g\}},
$$

where the bracket on the left hand side is the Jacobi-Lie bracket. Thus, it is natural to look at the corresponding algebra of Hamiltonian vector fields on the Poisson manifold $\left(\mathbb{R}^{n},\{\cdot, \cdot\}_{N}\right)$ associated to quadratic Hamiltonians. If we take $f=Q_{X}$ and $g=Q_{Y}$, with $X_{f}=N X$ and $X_{g}=N Y$, and recall that the Jacobi-Lie bracket of linear vector fields is the negative of the commutator of the associated matrices, then we have the following result, which can also be verified directly.

Proposition 2.2 Equations (2.4) and (2.5) imply

$$
N[X, Y]_{N}=[N X, N Y] .
$$

Letting $\mathcal{L H}$ denote the Lie algebra of linear Hamiltonian vector fields on $\mathbb{R}^{n}$ relative to the commutator bracket of matrices, (2.6) states that the map

$$
X \in\left(\operatorname{Sym}(n, N),[\cdot, \cdot]_{N}\right) \mapsto N X \in(\mathcal{L H},[\cdot, \cdot])
$$

is a homomorphism of Lie algebras ${ }^{4}$.

Invertible Case. If $N$ is invertible, then this homomorphism is an isomorphism. In addition, the nondegeneracy of $N$ implies that $n$ is even and that $\mathbb{R}^{n}$ is a symplectic vector space relative to the symplectic form defined by $N^{-1}$, that is, $(\mathbf{u}, \mathbf{v}) \mapsto \mathbf{u} \cdot N^{-1} \mathbf{v}$ for $\mathbf{u}, \mathbf{v} \in \mathbb{R}^{n}$. Therefore, the Lie algebra $(\mathcal{L H},[\cdot, \cdot])$ is isomorphic to the Lie algebra $\mathfrak{s p}\left(n, N^{-1}\right)$ of linear infinitesimally symplectic maps of $\mathbb{R}^{n}$ relative to the symplectic form defined above by $N^{-1}$. Recall that elements $Z \in \mathfrak{s p}\left(n, N^{-1}\right)$ are characterized by the identity $Z^{T} N^{-1}+N^{-1} Z=0$ which is equivalent to the statement that $N^{-1} Z$ is a symmetric $n \times n$ matrix. Thus $N X \in \mathfrak{s p}\left(n, N^{-1}\right)$ is equivalent to $X=X^{T}$, as expected.

We summarize these considerations in the following statement that can also be found in [27] at the end of remark 22 in $\S 44$ page 245.

Proposition 2.3 Let $N \in \mathfrak{s o}(n)$. The $\operatorname{map} Q: X \in\left(\operatorname{Sym}(n, N),[\cdot, \cdot]_{N}\right) \mapsto Q_{X} \in\left(\mathcal{Q},\{\cdot, \cdot\}_{N}\right)$ is a Lie algebra isomorphism. The map $\Phi: X \in\left(\operatorname{Sym}(n, N),[\cdot, \cdot]_{N}\right) \mapsto N X \in(\mathcal{L H},[\cdot, \cdot])$ is a Lie algebra homomorphism and if $N$ is invertible it induces an isomorphism of $\left(\operatorname{Sym}(n, N),[\cdot, \cdot]_{N}\right)$ with $\mathfrak{s p}\left(n, N^{-1}\right)$.

Noninvertible Case. Assume that $N$ is a general skew-symmetric matrix, not necessarily invertible. We shall determine now the structure of the Lie algebra $\left(\operatorname{Sym}(n, N),[\cdot, \cdot]_{N}\right)$. The point of departure is the fact that if $N$ is non-degenerate, then $X \in\left(\operatorname{Sym}(n, N),[\cdot, \cdot]_{N}\right) \mapsto N X \in(\mathcal{L H},[\cdot, \cdot])=\left(\mathfrak{s p}\left(n, N^{-1}\right),[\cdot, \cdot]\right)$ is a Lie algebra isomorphism. Recall that if $\mathbb{R}^{n}$ has an inner product, which we shall take in what follows to be the usual dot product associated to the basis in which the skew-symmetric matrix $N$ is given, and $L: \mathbb{R}^{n} \rightarrow \mathbb{R}^{n}$ is a linear map, then $\mathbb{R}^{n}$ decomposes orthogonally as $\mathbb{R}^{n}=\operatorname{im} L^{T} \oplus \operatorname{ker} L$. Taking $L=N$ in this statement and recalling that $N^{T}=-N$, we get the orthogonal decomposition $\mathbb{R}^{n}=\operatorname{im} N \oplus \operatorname{ker} N$. Let $2 p=\operatorname{rank} N$ and $d:=n-2 p$. Then $\bar{N}:=\left.N\right|_{\operatorname{im} N}: \operatorname{im} N \rightarrow \operatorname{im} N$ defines a non-degenerate skew symmetric bilinear form and, by the previous proposition, $\left(\operatorname{Sym}(2 p),[\cdot, \cdot]_{\bar{N}}\right)$ is isomorphic as a Lie algebra to $\left(\mathfrak{s p}\left(2 p, \bar{N}^{-1}\right),[\cdot, \cdot]\right)$. In this direct sum decomposition of $\mathbb{R}^{n}$, the skew- symmetric matrix $N$ takes the form

$$
N=\left[\begin{array}{cc}
\bar{N} & 0 \\
0 & 0
\end{array}\right]
$$

\footnotetext{
${ }^{4}$ We thank Gopal Prasad for suggesting isomorphisms of this type; they are closely related to well-known properties of linear Hamiltonian vector fields, as in [16], Proposition 2.7.8.
} 
where $\bar{N}$ is a $(2 p) \times(2 p)$ skew-symmetric non-degenerate matrix.

The Lie algebra $\left(\operatorname{Sym}(2 p),[\cdot, \cdot]_{\bar{N}}\right)$ acts on the vector space $\mathcal{M}_{(2 p) \times d}$ of $(2 p) \times d$ matrices (which we can think of as linear maps of $\operatorname{ker} N$ to $\operatorname{im} N)$ by $S \cdot A:=S \bar{N} A$, where $S \in\left(\operatorname{Sym}(2 p),[\cdot, \cdot]_{\bar{N}}\right)$ and $A \in \mathcal{M}_{(2 p) \times d}$. Indeed, if $S, S^{\prime} \in \operatorname{Sym}(2 p)$ and $A \in \mathcal{M}_{(2 p) \times d}$, then

$$
\begin{aligned}
{\left[S, S^{\prime}\right]_{\bar{N}} \cdot A } & =\left(S \bar{N} S^{\prime}-S^{\prime} \bar{N} S\right) \bar{N} A=S \bar{N} S^{\prime} \bar{N} A-S^{\prime} \bar{N} S \bar{N} A \\
& =S \cdot\left(S^{\prime} \cdot A\right)-S^{\prime} \cdot(S \cdot A) .
\end{aligned}
$$

Now form the semidirect product $\operatorname{Sym}(2 p) \subseteq \mathcal{M}_{(2 p) \times d}$. Its bracket is defined by

$$
\begin{aligned}
{\left[(S, A),\left(S^{\prime}, A^{\prime}\right)\right] } & =\left(\left[S, S^{\prime}\right]_{\bar{N}}, S \cdot A^{\prime}-S^{\prime} \cdot A\right) \\
& =\left(S \bar{N} S^{\prime}-S^{\prime} \bar{N} S, S \bar{N} A^{\prime}-S^{\prime} \bar{N} A\right)
\end{aligned}
$$

for any $S, S^{\prime} \in \operatorname{Sym}(2 p)$ and $A, A^{\prime} \in \mathcal{M}_{(2 p) \times d}$.

Next, define the $\operatorname{Sym}(d)$-valued Lie algebra two-cocycle

$$
C: \operatorname{Sym}(2 p) \subseteq \mathcal{M}_{(2 p) \times d} \times \operatorname{Sym}(2 p) \Subset \mathcal{M}_{(2 p) \times d} \rightarrow \operatorname{Sym}(d)
$$

by

$$
C\left((S, A),\left(S^{\prime}, A^{\prime}\right)\right):=A^{T} \bar{N} A^{\prime}-\left(A^{\prime}\right)^{T} \bar{N} A
$$

for any $S, S^{\prime} \in \operatorname{Sym}(2 p)$ and $A, A^{\prime} \in \mathcal{M}_{(2 p) \times d}$. The cocycle identity

$$
\begin{aligned}
& C\left(\left[(S, A),\left(S^{\prime}, A^{\prime}\right)\right],\left(S^{\prime \prime}, A^{\prime \prime}\right)\right)+C\left(\left[\left(S^{\prime}, A^{\prime}\right),\left(S^{\prime \prime}, A^{\prime \prime}\right)\right],(S, A)\right) \\
& \quad+C\left(\left[\left(S^{\prime \prime}, A^{\prime \prime}\right),(S, A)\right],\left(S^{\prime}, A^{\prime}\right)\right)=0
\end{aligned}
$$

for any $S, S^{\prime}, S^{\prime \prime} \in \operatorname{Sym}(2 p)$ and $A, A^{\prime}, A^{\prime \prime} \in \mathcal{M}_{(2 p) \times d}$ is a straightforward verification. Now extend $\operatorname{Sym}(2 p) \subseteq \mathcal{M}_{(2 p) \times d}$ by this cocycle. That is, form the vector space $\left(\operatorname{Sym}(2 p) \subseteq \mathcal{M}_{(2 p) \times d}\right) \oplus \operatorname{Sym}(d)$ and endow it with the bracket

$$
\begin{gathered}
{\left[(S, A, B),\left(S^{\prime}, A^{\prime}, B^{\prime}\right)\right]^{C}:=\left(S \bar{N} S^{\prime}-S^{\prime} \bar{N} S, S \bar{N} A^{\prime}-S^{\prime} \bar{N} A\right.} \\
\left.A^{T} \bar{N} A^{\prime}-\left(A^{\prime}\right)^{T} \bar{N} A\right)
\end{gathered}
$$

for any $S, S^{\prime} \in \operatorname{Sym}(2 p), A, A^{\prime} \in \mathcal{M}_{(2 p) \times d}$, and $B, B^{\prime} \in \operatorname{Sym}(d)$.

Proposition 2.4 The map

$$
\Psi:\left(\left(\operatorname{Sym}(2 p) \subseteq \mathcal{M}_{(2 p) \times d}\right) \oplus \operatorname{Sym}(d),[\cdot, \cdot]^{C}\right) \rightarrow\left(\operatorname{Sym}(n, N),[\cdot, \cdot]_{N}\right)
$$

given by

$$
\Psi(S, A, B):=\left[\begin{array}{cc}
S & A \\
A^{T} & B
\end{array}\right]
$$

is a Lie-algebra isomorphism.

Proof. It is obvious that $\Psi$ is a vector space isomorphism, therefore only the Lie-algebra homomorphism condition needs to be verified. So, let $(S, A, B),\left(S^{\prime}, A^{\prime}, B^{\prime}\right) \in\left(\operatorname{Sym}(2 p) \subseteq \mathcal{M}_{(2 p) \times d}\right) \oplus \operatorname{Sym}(d)$ and compute

$$
\begin{aligned}
\Psi\left(\left[(S, A, B),\left(S^{\prime}, A^{\prime}, B^{\prime}\right)\right]\right)=\Psi\left(S \bar{N} S^{\prime}-S^{\prime} \bar{N} S, S \bar{N} A^{\prime}-S^{\prime} \bar{N} A, A^{T} \bar{N} A^{\prime}-\left(A^{\prime}\right)^{T} \bar{N} A\right) \\
\quad=\left[\begin{array}{cc}
S \bar{N} S^{\prime}-S^{\prime} \bar{N} S & S \bar{N} A^{\prime}-S^{\prime} \bar{N} A \\
\left(S \bar{N} A^{\prime}-S^{\prime} \bar{N} A\right)^{T} & A^{T} \bar{N} A^{\prime}-\left(A^{\prime}\right)^{T} \bar{N} A
\end{array}\right] \\
\quad=\left[\begin{array}{cc}
S & A \\
A^{T} & B
\end{array}\right]\left[\begin{array}{cc}
\bar{N} & 0 \\
0 & 0
\end{array}\right]\left[\begin{array}{cc}
S^{\prime} & A^{\prime} \\
\left(A^{\prime}\right)^{T} & B^{\prime}
\end{array}\right]-\left[\begin{array}{cc}
S^{\prime} & A^{\prime} \\
\left(A^{\prime}\right)^{T} & B^{\prime}
\end{array}\right]\left[\begin{array}{cc}
\bar{N} & 0 \\
0 & 0
\end{array}\right]\left[\begin{array}{cc}
S & A \\
A^{T} & B
\end{array}\right] \\
\quad=\left[\Psi(S, A, B), \Psi\left(S^{\prime}, A^{\prime}, B^{\prime}\right)\right]_{N}
\end{aligned}
$$

as required.

For a different description of the structure of this Lie algebra using its Levi decomposition and not involving cocycles see [27], §44, Remark 22, page 245. 
Euler-Poincaré Form. The Euler-Poincaré form for the equations can be derived as follows. Identify $\operatorname{Sym}(n, N)$ with its dual using the the positive definite inner product

$$
\langle\langle X, Y\rangle:=\operatorname{trace}(X Y), \quad \text { for } \quad X, Y \in \operatorname{Sym}(n, N) .
$$

Remark. The inner product $\langle\langle X, Y\rangle\rangle$ is not ad-invariant relative to the $N$-bracket, but the bilinear form

$$
\kappa_{N}(X, Y):=\operatorname{trace}(N X N Y),
$$

is invariant, as is easy to check. Note that for $N$ invertible $\kappa_{N}$ is non-degenerate and hence an inner product and provides another method of identifying $\operatorname{Sym}(n)$ with its dual. We shall return to this observation at the end of $\S 4$.

Define the Lagrangian $l: \operatorname{Sym}(n, N) \rightarrow \mathbb{R}$ on the Lie algebra $\left(\operatorname{Sym}(n, N),[\cdot, \cdot]_{N}\right)$ by

$$
l(X)=\frac{1}{2} \operatorname{trace}\left(X^{2}\right)=\frac{1}{2} \operatorname{trace}\left(X X^{T}\right)=\frac{1}{2}\langle\langle X, X\rangle\rangle .
$$

Proposition 2.5 The equations

$$
\dot{X}=\left[X^{2}, N\right]
$$

are the Euler-Poincaré equations ${ }^{5}$ corresponding to the Lagrangian $(2.14)$ on the Lie algebra $\left(\operatorname{Sym}(n, N),[\cdot, \cdot]_{N}\right)$.

Proof. Recall that the general (left) Euler-Poincaré equations on a Lie algebra $\mathfrak{g}$ associated with a Lagrangian $l: \mathfrak{g} \rightarrow \mathbb{R}$ are given by

$$
\frac{d}{d t} D l(\xi)=\operatorname{ad}_{\xi}^{*} D l(\xi),
$$

where $D l(\xi) \in \mathfrak{g}^{*}$ is the Fréchet derivative of $l$ at $\xi$. Equivalently, for each fixed $\eta \in \mathfrak{g}$, we have

$$
\frac{d}{d t} D l(\xi) \cdot \eta=D l(\xi) \cdot[\xi, \eta] .
$$

In our case, letting $\xi=X$ and $\eta=Y$ arbitrary, time-independent, equations (2.16) become

$$
\begin{aligned}
\frac{d}{d t}\langle\langle X, Y\rangle\rangle & =\left\langle\left\langle X,[X, Y]_{N}\right\rangle\right\rangle \\
& =\langle\langle X, X N Y-Y N X\rangle\rangle ;
\end{aligned}
$$

that is,

$$
\begin{aligned}
\operatorname{trace}(\dot{X} Y) & =\operatorname{trace}(X(X N Y-Y N X)) \\
& =\operatorname{trace}\left(\left(X^{2} N-N X^{2}\right) Y\right)
\end{aligned}
$$

which gives the result.

\section{Poisson Structures}

Two compatible Poisson structures on $\operatorname{Sym}(n, N)$ are introduced in this section. Their associated Poisson geometry is studied in detail. These two structures together with the bi-Hamiltonian methodology will be the key to proving integrability of (1.1).

\footnotetext{
${ }^{5}$ For a general discussion of the Euler-Poincaré equations, see, for instance, [16].
} 
Two Poisson Structures. Identifying $\operatorname{Sym}(n, N)$ with its dual using the inner product $\langle\langle\cdot, \cdot\rangle\rangle$ defined in (2.12), endows $\operatorname{Sym}(n, N)$ with the the (left, or minus) Lie-Poisson bracket

$$
\{f, g\}_{N}(X)=-\operatorname{trace}[X(\nabla f(X) N \nabla g(X)-\nabla g(X) N \nabla f(X))],
$$

where $\nabla f$ is the gradient of $f$ relative to the inner product $\langle\langle\cdot, \cdot\rangle\rangle$ on $\operatorname{Sym}(n, N)$.

Later on we shall also need the frozen Poisson bracket

$$
\{f, g\}_{F N}(X)=-\operatorname{trace}(\nabla f(X) N \nabla g(X)-\nabla g(X) N \nabla f(X)) .
$$

It is a general fact that the Poisson structures (3.1) and (3.2) are compatible in the sense that their sum is a Poisson structure (see e.g. Exercise 10.1-5 in [16]).

For what follows it is important to compute the Poisson tensors corresponding to the above Poisson brackets. Recall that the Poisson tensor can be viewed as a vector bundle morphism $B: T^{*}(\operatorname{Sym}(n, N)) \rightarrow$ $T(\operatorname{Sym}(n, N))$ covering the identity. It is defined by $B(\mathbf{d} h)=\{\cdot, h\}_{N}$ for any locally defined smooth function $h$ on $\operatorname{Sym}(n, N)$. Since $\operatorname{Sym}(n, N)$ is a vector space, these bundles are trivial and hence the value $B_{X}$ at $X \in \operatorname{Sym}(n, N)$ of the Poisson tensor $B$ is a linear map $B_{X}: \operatorname{Sym}(n, N) \rightarrow \operatorname{Sym}(n, N)$ by identifying $\operatorname{Sym}(n, N)$ with its dual using the inner product $\langle\langle\cdot, \cdot\rangle\rangle$.

Proposition 3.1 Denote the value at $X \in \operatorname{Sym}(n, N)$ of the Poisson tensors corresponding to the LiePoisson (3.1) and frozen (3.2) brackets by $B_{X}$ and $C_{X}$, respectively. Then for any $Y \in \operatorname{Sym}(n, N)$ we have

$$
\begin{aligned}
& B_{X}(Y)=X Y N-N Y X \\
& C_{X}(Y)=Y N-N Y .
\end{aligned}
$$

Proof. Let $f$ and $g$ be locally defined smooth functions on $\operatorname{Sym}(n, N)$. The definition of $B_{X}$ gives

$$
\begin{aligned}
\left\langle\left\langle\nabla f(X), B_{X}(\nabla g(X)\rangle\right\rangle\right. & =\{f, g\}_{N}(X) \\
& =-\operatorname{trace}[X(\nabla f(X) N \nabla g(X)-\nabla g(X) N \nabla f(X))] \\
& =\operatorname{trace}[\nabla f(X)(X \nabla g(X) N-N \nabla g(X) X)] \\
& =\langle\langle\nabla f(X), X \nabla g(X) N-N \nabla g(X) X\rangle,
\end{aligned}
$$

which implies (3.3) since any $Y \in \operatorname{Sym}(n, N)$ is of the form $\nabla g(X)$, where $g(X)=\langle\langle X, Y\rangle\rangle$. Similarly, the definition of $C_{X}$ gives

$$
\begin{aligned}
\left\langle\left\langle\nabla f(X), C_{X}(\nabla g(X)\rangle\right\rangle\right. & =\{f, g\}_{F N}(X) \\
& =-\operatorname{trace}(\nabla f(X) N \nabla g(X)-\nabla g(X) N \nabla f(X)) \\
& =\operatorname{trace}[\nabla f(X)(\nabla g(X) N-N \nabla g(X))] \\
& =\langle\langle\nabla f(X), \nabla g(X) N-N \nabla g(X)\rangle,
\end{aligned}
$$

which proves (3.4).

Hamiltonian Vector Fields. Let us determine the Hamiltonian vector fields associated to a smooth function for both Poisson brackets. Recall that if $\mathfrak{g}$ is a Lie algebra, the Lie-Poisson equations defined by $h \in C^{\infty}\left(\mathfrak{g}^{*}\right)$ relative to the minus Lie-Poisson bracket are

$$
\dot{\mu}=\operatorname{ad}_{\delta h / \delta \mu}^{*} \mu
$$

where $\mu \in \mathfrak{g}^{*}$. 
We shall identify $\operatorname{Sym}(n, N)^{*}$ with itself via the inner product $\langle\langle\cdot, \cdot\rangle\rangle$. Therefore, for any $X, Y, Z \in$ $\operatorname{Sym}(n, N)$, we have

$$
\begin{aligned}
\left\langle\left\langle\left(\operatorname{ad}_{Y}^{N}\right)^{*} X, Z\right\rangle\right\rangle & =\left\langle\left\langle X,[Y, Z]_{N}\right\rangle\right\rangle=\operatorname{trace}(X Y N Z-X Z N Y) \\
& =\operatorname{trace}((X Y N-N Y X) Z)=\langle\langle X Y N-N Y X, Z\rangle\rangle
\end{aligned}
$$

and hence

$$
\left(\operatorname{ad}_{Y}^{N}\right)^{*} X=X Y N-N Y X
$$

If $h \in C^{\infty}(\operatorname{Sym}(n, N))$, we denote by $\nabla h(X)$ the gradient relative to the inner product $\langle\langle$,$\rangle . Therefore, the$ Lie-Poisson equations for $h \in C^{\infty}(\operatorname{Sym}(n, N))$ are

$$
\dot{X}=\left(\operatorname{ad}_{\nabla h(X)}^{N}\right)^{*} X,
$$

that is,

$$
\dot{X}=X \nabla h(X) N-N \nabla h(X) X .
$$

Similarly, Hamilton's equations for the frozen bracket are

$$
\dot{X}=\nabla h(X) N-N \nabla h(X) .
$$

In particular, if $h(X)=\operatorname{trace}\left(X^{2}\right) / 2$, equation (3.5) becomes $\dot{X}=\left[X^{2}, N\right]$. Similarly, if $h(X)=\operatorname{trace}\left(X^{3}\right) / 3$, equation (3.6) becomes $\dot{X}=\left[X^{2}, N\right]$.

If $N$ is invertible, we have seen that there is an ad-invariant inner product $\kappa_{N}(X, Y)=\operatorname{trace}(N X N Y)$. Therefore, we can identify $\operatorname{Sym}(n, N)^{*}$ with itself using the inner product $\kappa_{N}$. Denote by $\left(\operatorname{ad}_{Y}^{N}\right)^{\dagger}$ the

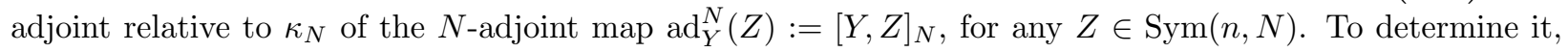
let $M, Y, Z \in \operatorname{Sym}(n, N)$ be arbitrary ( $M$ thought of as an element in the dual), compute

$$
\begin{aligned}
\kappa_{N}\left(\left(\operatorname{ad}_{Y}^{N}\right)^{\dagger} M, Z\right) & =\kappa_{N}\left(M,[Y, Z]_{N}\right)=\operatorname{trace}(N M N(Y N Z-Z N Y)) \\
& =\operatorname{trace}(N(M N Y-Y N M) N Z)=\kappa_{N}((M N Y-Y N M), Z),
\end{aligned}
$$

and conclude that

$$
\left(\operatorname{ad}_{Y}^{N}\right)^{\dagger} M=M N Y-Y N M=[M, Y]_{N} .
$$

If $h \in C^{\infty}(\operatorname{Sym}(n, N))$, denote by $\nabla^{N} h(M)$ the gradient relative to the inner product $\kappa_{N}$. Therefore, the Lie-Poisson equations for $h \in C^{\infty}(\operatorname{Sym}(n, N))$ are

$$
\dot{M}=\left(\operatorname{ad}_{\nabla^{N} h(M)}^{N}\right)^{\dagger} M=\left[M, \nabla^{N} h(M)\right]_{N} .
$$

For example, if $h(M)=\operatorname{trace}\left(N^{2} M N^{2} M\right) / 2$, then for any $S \in \operatorname{Sym}(n, N)$ we get

$$
\begin{aligned}
\operatorname{trace}\left(N^{2} M N^{2} S\right) & =\mathbf{d} h(M) \cdot S=\kappa_{N}\left(\nabla^{N} h(M), S\right) \\
& =\operatorname{trace}\left(N \nabla^{N} h(M) N S\right)
\end{aligned}
$$

and hence

$$
\nabla^{N} h(M)=N M N
$$

so Hamilton's equations (3.7) are

$$
\dot{M}=[M, N M N]_{N} .
$$

Note that if $l(X)=\langle\langle X, X\rangle\rangle / 2=\operatorname{trace}\left(X^{2}\right) / 2$ then the Legendre transform $M:=\nabla^{N} l(X)=N^{-1} X N^{-1}$ gives the Hamiltonian

$$
h(M):=\kappa_{N}(M, X)-l(X)=\frac{1}{2} \operatorname{trace}\left(N^{2} M N^{2} M\right) .
$$

Hence the Lie-Poisson equation (3.8) is equivalent to the Euler-Poicaré equation (2.15). One can check this fact explicitly: substituting for $M$ in terms of $X$ in (3.8) gives (2.15) and vice versa. 
Generic Leaves. Next, the dimension of the generic leaves of the two Poisson brackets are determined. The Lie-Poisson bracket is treated first. The following Proposition follows from [27], §44, Proposition 23, page 245. We give below an elementary proof.

Proposition 3.2 Let $n=2 p+d$, where $2 p=\operatorname{rank} N$. The generic leaves of the Lie-Poisson bracket $\{\cdot, \cdot\}_{N}$ are $2 p(p+d)$-dimensional.

Proof. As in the proof of Proposition 2.4, we orthogonally decompose $\mathbb{R}^{n}=\operatorname{im} N \oplus \operatorname{ker} N$ so that $\bar{N}=$ $N \mid \operatorname{im} N: \operatorname{im} N \rightarrow \operatorname{im} N$ is an isomorphism. In this decomposition the matrix $N$ takes the form

$$
N=\left[\begin{array}{cc}
\bar{N} & 0 \\
0 & 0
\end{array}\right]
$$

and, according to the isomorphism $\Psi$ in Proposition 2.4, the matrix $X$ can be written as

$$
X=\left[\begin{array}{cc}
S & A \\
A^{T} & B
\end{array}\right]
$$

where $S \in \operatorname{Sym}(2 p), B \in \operatorname{Sym}(d)$, and $A \in \mathcal{M}_{(2 p) \times d}$. Therefore, if

$$
Y=\left[\begin{array}{cc}
U & C \\
C^{T} & D
\end{array}\right] \in \operatorname{Sym}(n, N)
$$

with $U \in \operatorname{Sym}(2 p), D \in \operatorname{Sym}(d), C \in \mathcal{M}_{(2 p) \times d}$, the Poisson tensor of the Lie-Poisson bracket $\{\cdot, \cdot\}_{N}$ takes the form (see Proposition 3.1)

$$
\begin{aligned}
B_{X}(Y) & =X Y N-N Y X \\
& =\left[\begin{array}{cc}
S & A \\
A^{T} & B
\end{array}\right]\left[\begin{array}{cc}
U & C \\
C^{T} & D
\end{array}\right]\left[\begin{array}{cc}
\bar{N} & 0 \\
0 & 0
\end{array}\right]-\left[\begin{array}{cc}
\bar{N} & 0 \\
0 & 0
\end{array}\right]\left[\begin{array}{cc}
U & C \\
C^{T} & D
\end{array}\right]\left[\begin{array}{cc}
S & A \\
A^{T} & B
\end{array}\right] \\
& =\left[\begin{array}{cc}
S U \bar{N}-\bar{N} U S+A C^{T} \bar{N}-\bar{N} C A^{T} & -\bar{N} U A-\bar{N} C B \\
A^{T} U \bar{N}+B C^{T} \bar{N} & 0
\end{array}\right] .
\end{aligned}
$$

Since $\bar{N}$ is invertible, the kernel of $B_{X}: \operatorname{Sym}(n, N) \rightarrow \operatorname{Sym}(n, N)$ is therefore given by all $U \in \operatorname{Sym}(2 p)$, $D \in \operatorname{Sym}(d)$, and $C \in \mathcal{M}_{(2 p) \times d}$ such that

$$
S U \bar{N}-\bar{N} U S+A C^{T} \bar{N}-\bar{N} C A^{T}=0 \quad \text { and } \quad U A+C B=0 .
$$

To compute the dimension of the maximal symplectic leaves, we assume that the matrix $X$ is generic. So, supposing that $B$ is invertible, we have $C=-U A B^{-1}$ and

$$
\left(S-A B^{-1} A^{T}\right) U \bar{N}-\bar{N} U\left(S-A B^{-1} A^{T}\right)=0 .
$$

Since $S-A B^{-1} A^{T} \in \operatorname{Sym}(2 p)$ is given, this condition is identical to the vanishing of the Poisson tensor on the dual of the Lie algebra $\left(\operatorname{Sym}(2 p, \bar{N}),[\cdot, \cdot]_{\bar{N}}\right)$ evaluated at $S-A B^{-1} A^{T}$. But $\bar{N}$ is invertible so, according to Proposition 2.3, this Lie algebra is isomorphic to $\mathfrak{s p}\left(2 p, \bar{N}^{-1}\right)$ whose rank is $p$. Therefore, the kernel of the map

$$
U \in \operatorname{Sym}(2 p, \bar{N}) \mapsto\left(S-A B^{-1} A^{T}\right) U \bar{N}-\bar{N} U\left(S-A B^{-1} A^{T}\right) \in \operatorname{Sym}(2 p, \bar{N})
$$

for generic $S-A B^{-1} A^{T}$ has dimension $p$.

Since $C=-U A B^{-1}$ is uniquely determined and $D \in \operatorname{Sym}(d)$ is arbitrary, we see that the dimension of the kernel of $B_{X}$ for generic $X$ has dimension $p+d(d+1) / 2$.

Thus, the dimension of the generic leaf of the Lie-Poisson bracket $\{\cdot, \cdot\}_{N}$ is

$$
\frac{1}{2}(2 p+d)(2 p+d+1)-p-\frac{1}{2} d(d+1)=2 p(p+d)
$$

as claimed in the statement of the proposition. 
Proposition 3.3 All the leaves of the frozen Poisson bracket $\{\cdot, \cdot\}_{F N}$ are

(i) $2 p(p+d)$-dimensional if $N$ is generic, that is, all its non-zero eigenvalues are distinct, and

(ii) $p(p+1+2 d)$-dimensional if all non-zero eigenvalue pairs of $N$ are equal.

Proof. Proceeding as in the proof of the previous proposition and using the same notation for $N, X$, and $Y$, the Poisson tensor of the frozen bracket takes the form

$$
\begin{aligned}
C_{X}(Y) & =Y N-N Y=\left[\begin{array}{cc}
U & C \\
C^{T} & D
\end{array}\right]\left[\begin{array}{cc}
\bar{N} & 0 \\
0 & 0
\end{array}\right]-\left[\begin{array}{cc}
\bar{N} & 0 \\
0 & 0
\end{array}\right]\left[\begin{array}{cc}
U & C \\
C^{T} & D
\end{array}\right] \\
& =\left[\begin{array}{cc}
U \bar{N}-\bar{N} U & \bar{N} C \\
C^{T} \bar{N} & 0
\end{array}\right] .
\end{aligned}
$$

Thus, since $\bar{N}$ is invertible, the kernel of $C_{X}$ is given by all $U \in \operatorname{Sym}(2 p), D \in \operatorname{Sym}(d), C \in \mathcal{M}_{(2 p) \times d}$ such that $C=0$ and $U \bar{N}-\bar{N} U=0$.

Since $\bar{N}$ is non-degenerate, there exists an orthogonal matrix $Q$ such that

$$
\bar{N}=Q^{T}\left[\begin{array}{cc}
0 & V \\
-V & 0
\end{array}\right] Q
$$

where $V=\operatorname{diag}\left(v_{1}, \ldots, v_{p}\right)$ and $v_{i} \in \mathbb{R}, v_{i} \neq 0$ for all $i=1, \ldots, p$. Therefore,

$$
\begin{aligned}
0 & =U \bar{N}-\bar{N} U=U Q^{T}\left[\begin{array}{cc}
0 & V \\
-V & 0
\end{array}\right] Q-Q^{T}\left[\begin{array}{cc}
0 & V \\
-V & 0
\end{array}\right] Q U \\
& =Q^{T}\left(Q U Q^{T}\left[\begin{array}{cc}
0 & V \\
-V & 0
\end{array}\right]-\left[\begin{array}{cc}
0 & V \\
-V & 0
\end{array}\right] Q U Q^{T}\right) Q
\end{aligned}
$$

is equivalent to

$$
\tilde{U}\left[\begin{array}{cc}
0 & V \\
-V & 0
\end{array}\right]-\left[\begin{array}{cc}
0 & V \\
-V & 0
\end{array}\right] \tilde{U}=0
$$

where $\tilde{U}:=Q U Q^{T} \in \operatorname{Sym}(2 p)$. Write

$$
\tilde{U}=\left[\begin{array}{ll}
U_{11} & U_{12} \\
U_{12}^{T} & U_{22}
\end{array}\right]
$$

with $U_{11}$ and $U_{22}$ symmetric $p \times p$ matrices and $U_{12}$ an arbitrary $p \times p$ matrix. Then (3.9) is equivalent to

$$
U_{22}=V U_{11} V^{-1}=V^{-1} U_{11} V \text { and } U_{12}^{T}=-V^{-1} U_{12} V=-V U_{12} V^{-1} .
$$

(i) Assume now that $v_{i} \neq v_{j}$ if $i \neq j$. Since $V U_{11} V^{-1}=V^{-1} U_{11} V$ is equivalent to $V^{2} U_{11} V^{-2}=U_{11}$, it follows that

$$
\frac{v_{i}^{2}}{v_{j}^{2}} u_{11, i j}=u_{11, i j} \quad \text { for all } i, j=1, \ldots, p,
$$

where $u_{11, i j}$ are the entries of the symmetric matrix $U_{11}$. Since the fraction on the left hand side is never equal to one for $i \neq j$, this relation implies that $u_{11, i j}=0$ for all $i \neq j$. Thus $U_{11}$ is diagonal and $U_{22}=U_{11}$. A similar argument shows that $U_{12}$ is diagonal. However, then it follows that $U_{12}=-U_{12}^{T}$ which implies that $U_{12}=0$. Therefore, the kernel of the map $U \mapsto U \bar{N}-\bar{N} U$ is $p$-dimensional.

Concluding, the dimension of every leaf of the frozen Poisson structure equals $\frac{1}{2}(2 p+d)(2 p+d+1)-$ $p-\frac{1}{2} d(d+1)=2 p(p+d)$.

(ii) The other extreme case is when $v_{i}=v_{j}=: v$ for all $i, j=1, \ldots, p$. Then $V=v I$, where $I$ is the identity matrix, and (3.10) becomes $U_{22}=U_{11}, U_{12}^{T}=-U_{12}$. Therefore, the kernel of the map $U \mapsto U \bar{N}-\bar{N} U$ has dimension equal to $\frac{1}{2} p(p+1)+\frac{1}{2} p(p-1)=p^{2}$.

Concluding, the dimension of every leaf of the frozen Poisson structure equals $\frac{1}{2}(2 p+d)(2 p+d+1)-$ $p^{2}-\frac{1}{2} d(d+1)=p(p+1+2 d)$. 
Casimir Functions. The next job will be to determine Casimir functions for both brackets. Here is the main result.

Proposition 3.4 Let the skew symmetric matrix $N$ have rank $2 p$ and size $n:=2 p+d$. Choose an orthonormal basis of $\mathbb{R}^{2 p+d}$ in which $N$ is written as

$$
N=\left[\begin{array}{ccc}
0 & V & 0 \\
-V & 0 & 0 \\
0 & 0 & 0
\end{array}\right]
$$

where $V$ is a real diagonal matrix whose entries are $v_{1}, \ldots, v_{p}$.

(i) If $v_{i} \neq v_{j}$ for all $i \neq j$, then $p+d(d+1) / 2$ Casimir functions for the frozen Poisson structure (3.2) are given by

$$
C_{F}^{i}(X)=\operatorname{trace}\left(E_{i} X\right), \quad i=1, \ldots, p+\frac{1}{2} d(d+1),
$$

where $E_{i}$ is any of the matrices

$$
\left[\begin{array}{ccc}
S_{k k} & 0 & 0 \\
0 & S_{k k} & 0 \\
0 & 0 & 0
\end{array}\right], \quad\left[\begin{array}{ccc}
0 & 0 & 0 \\
0 & 0 & 0 \\
0 & 0 & S_{a b}
\end{array}\right]
$$

Here $S_{k k}$ is the $p \times p$ matrix all of whose entries are zero except the diagonal $(k, k)$ entry which is one and $S_{a b}$ is the $d \times d$ symmetric matrix having all entries equal to zero except for the $(a, b)$ and $(b, a)$ entries that are equal to one.

(ii) If $v_{i}=v_{j}$ for all $i, j=1, \ldots, p$, then $p^{2}+d(d+1) / 2$ Casimir functions for the frozen Poisson structure (3.2) are given by

$$
C_{F}^{i}(X)=\operatorname{trace}\left(E_{i} X\right), \quad i=1, \ldots, p^{2}+\frac{1}{2} d(d+1),
$$

where $E_{i}$ is any of the matrices

$$
\left[\begin{array}{ccc}
S_{k l} & 0 & 0 \\
0 & S_{k l} & 0 \\
0 & 0 & 0
\end{array}\right], \quad\left[\begin{array}{ccc}
0 & A_{k l} & 0 \\
-A_{k l} & 0 & 0 \\
0 & 0 & 0
\end{array}\right], \quad\left[\begin{array}{ccc}
0 & 0 & 0 \\
0 & 0 & 0 \\
0 & 0 & S_{a b}
\end{array}\right]
$$

Here $S_{k l}$ is the $p \times p$ symmetric matrix having all entries equal to zero except for the $(k, l)$ and $(l, k)$ entries that are equal to one and $A_{k l}$ is the $p \times p$ skew symmetric matrix with all entries equal to zero except for the $(k, l)$ entry which is 1 and the $(l, k)$ entry which is -1 .

(iii) Denote

$$
\bar{N}=\left[\begin{array}{cc}
0 & V \\
-V & 0
\end{array}\right]
$$

The $p+d(d+1) / 2$ Casimir functions for the Lie-Poisson bracket $\{\cdot, \cdot\}_{N}$ on the open set $\operatorname{det}(B) \neq 0$ (see (2.11)) of $\operatorname{Sym}(2 p+d)$ are given by

$$
C^{k}(X):=\frac{1}{2 k} \operatorname{trace}\left(\left[\left(S-A B^{-1} A^{T}\right) \bar{N}^{-1}\right]^{2 k}\right) . \quad \text { for } \quad k=1, \ldots, p
$$

and

$$
C^{k}(X)=\operatorname{trace}\left(X E_{k}\right), \quad \text { for } \quad k=p+1, \ldots, p+\frac{1}{2} d(d+1),
$$

where $E_{k}$ is any matrix of the form

$$
\left[\begin{array}{ccc}
0 & 0 & 0 \\
0 & 0 & 0 \\
0 & 0 & S_{a b}
\end{array}\right]
$$


In the special case when $N$ is full rank the Casimir functions are just

$$
C^{k}(X)=\frac{1}{2 k} \text { trace }\left[\left(X N^{-1}\right)^{2 k}\right], \quad \text { for } \quad k=1, \ldots, p,
$$

Proof. To prove (i), recall from Proposition 3.3(i) that the kernel of the Poisson tensor $C_{X}$ has dimension $p+\frac{1}{2} d(d+1)$. Moreover, if $E$ belongs to this kernel, then the linear function given by $X \mapsto \operatorname{trace}(E X)$ has gradient $E$, which is annihilated by the Poisson tensor $C_{X}$. Thus all $C_{F}^{i}$ are Casimir functions. Since the gradients of all these functions are the $p+\frac{1}{2} d(d+1)$ matrices in the statement which are obviously linearly independent, it follows that the functions $C_{F}^{i}$ form a functionally independent set of Casimir functions for the frozen bracket $\{\cdot, \cdot\}_{F N}$.

Part (ii) has an identical proof.

Now consider Part (iii). First, we compute the gradient relative to $\langle\langle\cdot, \cdot\rangle\rangle$. We compute for any

$$
\delta X=\left[\begin{array}{cc}
\delta S & \delta A \\
(\delta A)^{T} & \delta B
\end{array}\right] \in \operatorname{Sym}(n, N)
$$

the derivative

$$
\begin{gathered}
\mathbf{D} C^{k}(X) \cdot \delta X=\operatorname{trace}\left(\bar{N}^{-1}\left(S-A B^{-1} A^{T}\right) \bar{N}^{-1} \cdots \bar{N}^{-1}\left(S-A B^{-1} A^{T}\right) \bar{N}^{-1}\right. \\
\left.\left((\delta S)-(\delta A) B^{-1} A^{T}-A B^{-1}(\delta A)^{T}+A B^{-1}(\delta B) B^{-1} A^{T}\right)\right) .
\end{gathered}
$$

Now denote

$$
\nabla C^{k}(X)=\left[\begin{array}{cc}
\alpha & \beta \\
\beta^{T} & \gamma
\end{array}\right]
$$

so that

$$
\begin{aligned}
\operatorname{trace}\left(\nabla C^{k}(X)(\delta X)\right) & =\left\langle\left\langle\nabla C^{k}(X), \delta X\right\rangle\right\rangle=\operatorname{trace}\left(\left[\begin{array}{cc}
\alpha & \beta \\
\beta^{T} & \gamma
\end{array}\right]\left[\begin{array}{cc}
\delta S & \delta A \\
(\delta A)^{T} & \delta B
\end{array}\right]\right) \\
& =\operatorname{trace}\left(\alpha(\delta S)+\beta(\delta A)^{T}+\beta^{T}(\delta A)+\gamma(\delta B)\right) .
\end{aligned}
$$

By (3.11) and (3.12) we have

$$
\begin{aligned}
& \alpha=\bar{N}^{-1}\left(S-A B^{-1} A^{T}\right) \bar{N}^{-1} \cdots \bar{N}^{-1}\left(S-A B^{-1} A^{T}\right) \bar{N}^{-1} \\
& \beta=-\bar{N}^{-1}\left(S-A B^{-1} A^{T}\right) \bar{N}^{-1} \cdots \bar{N}^{-1}\left(S-A B^{-1} A^{T}\right) \bar{N}^{-1} A B^{-1} \\
& \gamma=B^{-1} A^{T} \bar{N}^{-1}\left(S-A B^{-1} A^{T}\right) \bar{N}^{-1} \cdots \bar{N}^{-1}\left(S-A B^{-1} A^{T}\right) \bar{N}^{-1} A B^{-1}
\end{aligned}
$$

where in each term we have $2 k$ factors of $\bar{N}^{-1}$. Therefore

$$
\nabla C^{k}(X)=\left[\begin{array}{cc}
\alpha & -\alpha A B^{-1} \\
-B^{-1} A^{T} \alpha & B^{-1} A^{T} \alpha A B^{-1}
\end{array}\right]
$$

with $\alpha$ given above. Now we check that all these matrices $\nabla C^{k}(X)$ are in the kernel of the operator of the Lie-Poisson operator $B_{X} Y=X Y N-N Y X$. Indeed,

$$
\begin{gathered}
X \nabla C^{k}(X) N-N \nabla C^{k}(X) X \\
=\left[\begin{array}{cr}
S & A \\
A^{T} & B
\end{array}\right]\left[\begin{array}{cc}
\alpha & -\alpha A B^{-1} \\
-B^{-1} A^{T} \alpha & B^{-1} A^{T} \alpha A B^{-1}
\end{array}\right]\left[\begin{array}{cc}
\bar{N} & 0 \\
0 & 0
\end{array}\right] \\
\quad-\left[\begin{array}{ll}
\bar{N} & 0 \\
0 & 0
\end{array}\right]\left[\begin{array}{cc}
\alpha & -\alpha A B^{-1} \\
-B^{-1} A^{T} \alpha & B^{-1} A^{T} \alpha A B^{-1}
\end{array}\right]\left[\begin{array}{cc}
S & A \\
A^{T} & B
\end{array}\right] \\
=\left[\begin{array}{cr}
S & A \\
A^{T} & B
\end{array}\right]\left[\begin{array}{cc}
\alpha \bar{N} & 0 \\
-B^{-1} A^{T} \alpha \bar{N} & 0
\end{array}\right]-\left[\begin{array}{cc}
\bar{N} \alpha & -\bar{N} \alpha A B^{-1} \\
0 & 0
\end{array}\right]\left[\begin{array}{cc}
S & A \\
A^{T} & B
\end{array}\right] \\
=\left[\begin{array}{cc}
S \alpha \bar{N}-A B^{-1} A^{T} \alpha \bar{N} & 0 \\
A^{T} \alpha \bar{N}-B B^{-1} A^{T} \alpha \bar{N} & 0
\end{array}\right]-\left[\begin{array}{cc}
\bar{N} \alpha S-\bar{N} \alpha A B^{-1} A^{T} & \bar{N} \alpha A-\bar{N} \alpha A B^{-1} B \\
0 & 0
\end{array}\right] \\
=\left[\begin{array}{cc}
\left(S-A B^{-1} A^{T}\right) \alpha \bar{N}-\bar{N} \alpha\left(S-A B^{-1} A^{T}\right) & 0 \\
0 & 0
\end{array}\right] .
\end{gathered}
$$


This vanishes if and only if

$$
\left(S-A B^{-1} A^{T}\right) \alpha \bar{N}-\bar{N} \alpha\left(S-A B^{-1} A^{T}\right)=0 .
$$

However, we know that $\alpha=\bar{N}^{-1}\left(S-A B^{-1} A^{T}\right) \bar{N}^{-1} \cdots \bar{N}^{-1}\left(S-A B^{-1} A^{T}\right) \bar{N}^{-1}$ where in each factor we have $2 k$ factors of $\bar{N}^{-1}$. We replace $\alpha$ with this expression in (3.13) and get

$$
\begin{aligned}
(S- & \left.A B^{-1} A^{T}\right) \alpha \bar{N}-\bar{N} \alpha\left(S-A B^{-1} A^{T}\right) \\
= & \left(S-A B^{-1} A^{T}\right) \bar{N}^{-1}\left(S-A B^{-1} A^{T}\right) \bar{N}^{-1} \cdots \bar{N}^{-1}\left(S-A B^{-1} A^{T}\right) \bar{N}^{-1} \bar{N} \\
& \quad-\bar{N} \bar{N}^{-1}\left(S-A B^{-1} A^{T}\right) \bar{N}^{-1} \cdots \bar{N}^{-1}\left(S-A B^{-1} A^{T}\right) \bar{N}^{-1}\left(S-A B^{-1} A^{T}\right) \\
= & \left(S-A B^{-1} A^{T}\right) \bar{N}^{-1}\left(S-A B^{-1} A^{T}\right) \bar{N}^{-1} \cdots \bar{N}^{-1}\left(S-A B^{-1} A^{T}\right) \\
& \quad-\left(S-A B^{-1} A^{T}\right) \bar{N}^{-1} \cdots \bar{N}^{-1}\left(S-A B^{-1} A^{T}\right) \bar{N}^{-1}\left(S-A B^{-1} A^{T}\right)=0
\end{aligned}
$$

since both factors are equal; each once contains $2 k-1$ factors of $\bar{N}^{-1}$.

However, $\mathfrak{s p}\left(2 p, \bar{N}^{-1}\right)$ is identified with the subalgebra consisting of the $(1,1)$ blocks of elements of $\operatorname{Sym}(n, N)$ (see Proposition 2.4). The isomorphism $S \in \operatorname{Sym}(2 p, \bar{N}) \mapsto \bar{N} S \in \mathfrak{s p}\left(2 p, \bar{N}^{-1}\right)$ given in Proposition 2.3 identifies the basis of $p$ Casimirs in the dual of $\mathfrak{s p}\left(2 p, \bar{N}^{-1}\right)$ (given by the even traces of the powers of a matrix) with the functions $S \mapsto \operatorname{trace}\left[\left(S \bar{N}^{-1}\right)^{2 k}\right] / 2 k$. Therefore the functions $C^{k}$ for $k=1, \ldots, p$ given in the statement of the proposition are functionally independent Casimirs for the Lie-Poisson bracket of $\operatorname{Sym}(n, N)$.

To see that the remaining functions $C^{k}(X)=\operatorname{trace}\left(X E_{k}\right)$ are Casimirs observe that in this case

$$
\nabla C^{k}(X)=\left[\begin{array}{cc}
0 & 0 \\
0 & S_{a b}
\end{array}\right]
$$

and

$$
B_{X}\left(\nabla C^{k}(X)\right)=\left[\begin{array}{cc}
S & A \\
A^{T} & B
\end{array}\right]\left[\begin{array}{cc}
0 & 0 \\
0 & S_{a b}
\end{array}\right]\left[\begin{array}{cc}
\bar{N} & 0 \\
0 & 0
\end{array}\right]-\left[\begin{array}{cc}
\bar{N} & 0 \\
0 & 0
\end{array}\right]\left[\begin{array}{cc}
0 & 0 \\
0 & S_{a b}
\end{array}\right]\left[\begin{array}{cc}
S & A \\
A^{T} & B
\end{array}\right]=0 .
$$

Since the matrices $S_{a b}$ span symmetric $k \times k$ matrices, these Casimirs are functionally independent. The two sets of Casimirs are also independent taken together, since each set depends only on a subset of independent variables and these two sets of variables are disjoint. We have thus obtained $p+d(d+1) / 2$ Casimirs, which is the codimension of the generic leaf, thus proving that they generate the space of all Casimir functions of the Lie-Poisson bracket.

The Equations in the Degenerate Case. If $N$ is degenerate, representing it and the matrix $X \in$ $\operatorname{Sym}(n, N)$ as in Proposition 2.4, the equations $\dot{X}=\left[X^{2}, N\right]$ are equivalent to the system

$$
\left\{\begin{array}{l}
\dot{S}=\left[S^{2}+A^{T} A, \bar{N}\right] \\
\dot{A}=-\bar{N}(S A+A B) \\
\dot{B}=0 .
\end{array}\right.
$$

Example. It is illuminating to examine the system in the lowest dimension degenerate case, i.e. $p=1$ and $d=1$. Let

$$
X=\left[\begin{array}{lll}
a & e & f \\
e & b & g \\
f & g & c
\end{array}\right]=:\left[\begin{array}{cc}
S & A \\
A^{T} & c
\end{array}\right]
$$

and

$$
N=\left[\begin{array}{ccc}
0 & 1 & 0 \\
-1 & 0 & 0 \\
0 & 0 & 0
\end{array}\right]=:\left[\begin{array}{cc}
\bar{N} & 0 \\
0 & 0
\end{array}\right]
$$


Then the dynamics becomes

$$
\begin{aligned}
\dot{a} & =-2(a e+e b+f g) \\
\dot{b} & =2(a e+e b+f g) \\
\dot{c} & =0 \\
\dot{e} & =a^{2}+f^{2}-b^{2}-g^{2} \\
\dot{g} & =a f+g e+c f \\
\dot{f} & =-(e f+b g+g c) .
\end{aligned}
$$

In this case the two Casimir functions of the Lie-Poisson bracket are given by

$$
C^{1}=\frac{1}{2}\left(-b a+\frac{g^{2} a}{c}+e^{2}-2 \frac{f g e}{c}+\frac{f^{2} b}{c}\right)=-\frac{\operatorname{det} X}{2 c}
$$

and by $C^{2}=c$, so that $\dot{c}=0$ in equations of motion expresses the conservation of this Casimir directly.

As we shall see in forthcoming sections the two integrals of motion which prove integrability are trace $(X)$ and trace $\left(X^{2}\right)$. We already know these are conserved since the flow is isospectral. Observe also that conservation of trace $(X)$ is given by summing the first two equations of motion while trace $(X)^{2} / 2$ is the Hamiltonian.

We illustrate this example with time plots in Figure 3.1 and two phase plots plots in Figure 3.2.

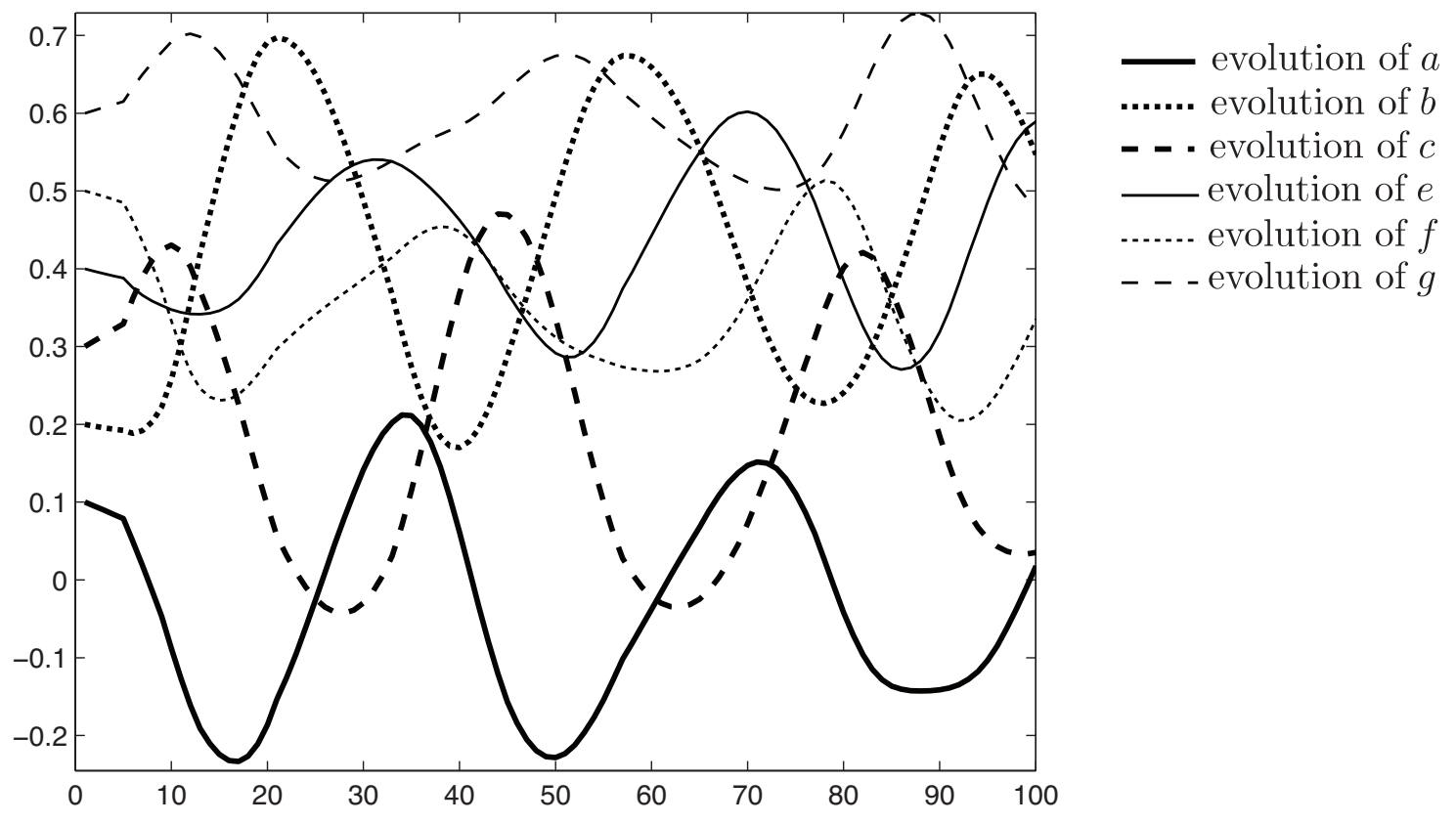

Figure 3.1: Time plot of flow in the 3 by 3 case for $a, b, c, e, f$, and $g$.

\section{The Sectional Operator Equations and Relation to Mischenko- Fomenko Flows}

It is shown that the equation (1.1) can be mapped to a Mischenko-Fomenko type system (see $[19 ; 20 ; 21]$ or [27]) in the case $N$ is invertible with distinct eigenvalues. 

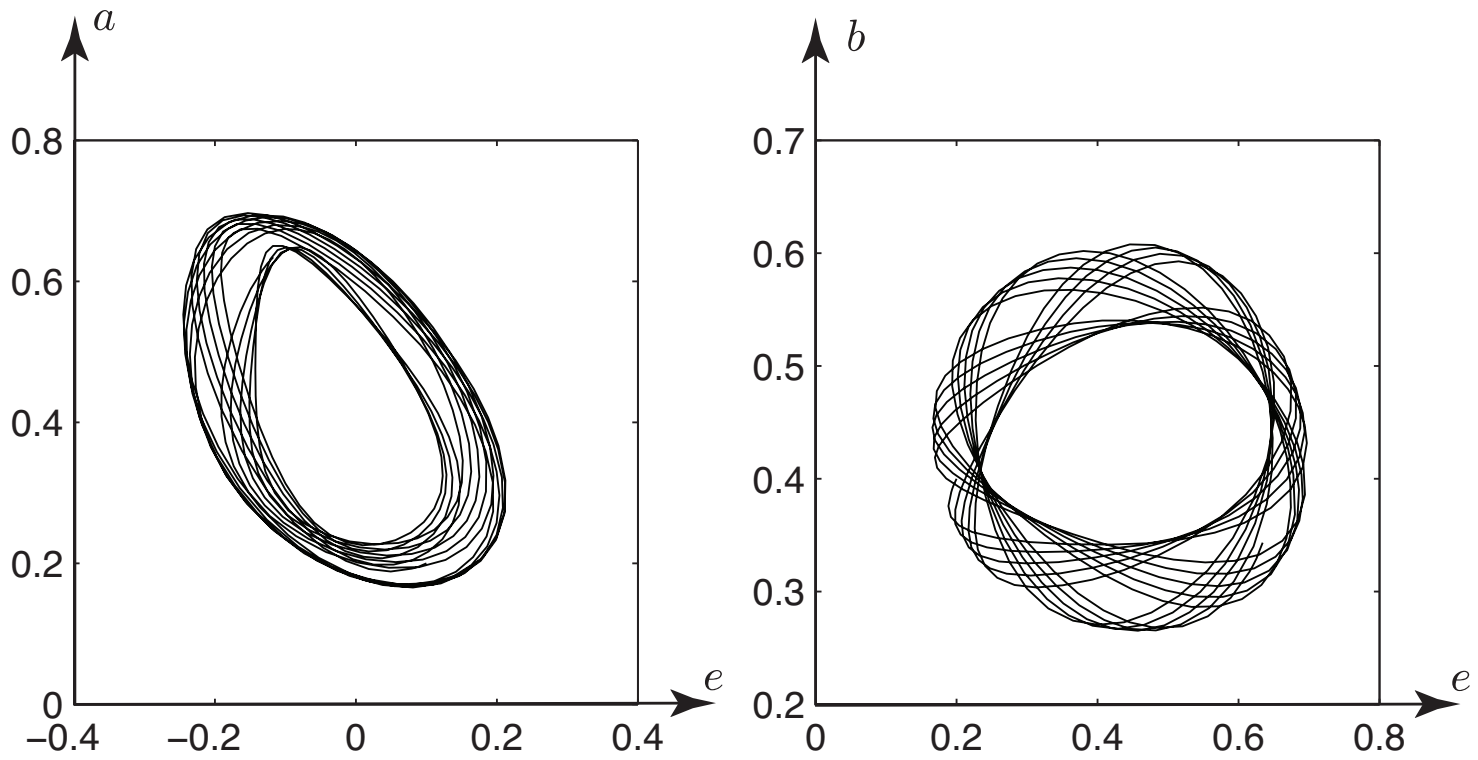

Figure 3.2: Phase plane portraits in the 3 by 3 case projected to the $a-e$ and the $b$-e planes.

The Mischenko-Fomenko Construction. Consider a semisimple complex or real split Lie algebra $\mathfrak{g}$ with Killing form $\langle\cdot, \cdot\rangle$. Let $\mathfrak{h}$ be a Cartan subalgebra, let $a, b \in \mathfrak{h}$ and $a$ be regular (i.e. its value on every root is non-zero). Define the sectional operators $C_{a, b, D}: \mathfrak{g} \rightarrow \mathfrak{g}$ by $C_{a, b, D}(\xi):=\operatorname{ad}_{a}^{-1} \operatorname{ad}_{b}\left(\xi_{1}\right)+D\left(\xi_{2}\right)$ where $\xi=\xi_{1}+\xi_{2}, \xi_{2} \in \mathfrak{h}, \xi_{1} \in \mathfrak{h}^{\perp}$ (the perpendicular is taken relative to the Killing form and thus $\mathfrak{h}^{\perp}$ is the direct sum of all the root spaces), and $D: \mathfrak{h} \rightarrow \mathfrak{h}$ is an arbitrary invertible symmetric operator on $\mathfrak{h}$. Then $C_{a, b, D}: \mathfrak{g} \rightarrow \mathfrak{g}$ is an invertible symmetric operator (relative to the Killing form) satisfying the condition

$$
\left[C_{a, b, D}(\xi), a\right]=[\xi, b]
$$

for all $\xi \in \mathfrak{g}$.

The Lie-Poisson bracket on $\mathfrak{g}^{*} \cong \mathfrak{g}$ (the isomorphism being given by the Killing form) has the expression

$$
\{f, g\}(\xi)=-\langle\xi,[\nabla f(\xi), \nabla g(\xi)]\rangle
$$

for any $f, g \in C^{\infty}(\mathfrak{g})$, where $\nabla$ is taken relative to $\langle\cdot, \cdot\rangle$. Hamilton's equations for $h \in C^{\infty}(\mathfrak{g})$ have thus the form

$$
\dot{\xi}=[\xi, \nabla h(\xi)] .
$$

In particular, if

$$
h(\xi):=\frac{1}{2}\left\langle C_{a, b, D}(\xi), \xi\right\rangle
$$

then $\nabla h(\xi)=C_{a, b, D}(\xi)$ since $C_{a, b, D}$ is $\langle\cdot, \cdot\rangle$-symmetric. Thus the equations of motion are

$$
\dot{\xi}=\left[\xi, C_{a, b, D}(\xi)\right] .
$$

Example: For $\mathfrak{g}=\mathfrak{s o}(n)$, the Killing form is a multiple of the symmetric bi-invariant two-form $\left(\Omega_{1}, \Omega_{2}\right) \mapsto$ $\operatorname{tr}\left(\Omega_{1} \Omega_{2}\right)$, and one chooses $C^{-1}(\Omega):=\Omega J+J \Omega$ for a given diagonal matrix $J$ satisfying $J_{i}+J_{j}>0$ if $i \neq j$. We have

$$
[C(M), J]=\left[M, J^{2}\right]
$$


for any $M \in \mathfrak{s o}(n)$. Then $\dot{M}=[M, C(M)]$ is the $n$-dimensional rigid body equation. Note in this case that $J$ and $J^{2}$ are not in the Cartan subalgebra of $\mathfrak{s o}(n)$, but the general theory in [20; 21] deals also with this situation for any semisimple complex or real split Lie algebra; $J$ an $J^{2}$ are in the Cartan subalgebra (after one makes them trace zero) of $\mathfrak{s l}(n, \mathbb{C})$.

Returning to the general case note that (4.2) can be written as

$$
\frac{d}{d t}(\xi+\lambda a)=[\xi+\lambda a, C(\xi)+\lambda b]
$$

if and only if (4.1) holds.

Now it is obvious that $\xi \mapsto f_{k}(\xi+\lambda a), k=1, \ldots, \ell:=\operatorname{rank}(\mathfrak{g})=\operatorname{dim} \mathfrak{h}$, are conserved on the flow of (4.3), for any element of the basis of the polynomial Casimir functions $f_{1}, \ldots, f_{\ell}$ and any parameter $\lambda$. Since the $f_{k}$ are polynomial, it follows that the coefficients of $\lambda^{i}$ in the expansion of $f_{k}(\xi+\lambda a)$ in powers of $\lambda$ are conserved along the flow of (4.2). There are redundancies: some coefficients of $\lambda^{i}$ vanish and other coefficients are Casimir functions.

Mischenko and Fomenko ([20;21]) proved the following result.

Theorem 4.1 Let $\mathfrak{g}$ be a semisimple complex or real split Lie algebra and $C: \mathfrak{g} \rightarrow \mathfrak{g}$ a symmetric operator satisfying (4.1). Then the Lie-Poisson system $\dot{\xi}=[\xi, C(\xi)]$ on $\mathfrak{g}$ defined by the Hamiltonian $H(\xi)=$ $\langle C(\xi), \xi\rangle / 2$ is completely integrable on the maximal dimensional adjoint orbits of the Lie algebra $\mathfrak{g}$ and its commuting generically independent first integrals are the non-trivial coefficients of $\lambda^{i}$ in the polynomial $\lambda$ expansion of

$$
f_{i, \lambda}(\xi)=f_{i}(\xi+\lambda a)
$$

which are not Casimir functions; here $f_{1}, \ldots, f_{\ell}$ is the basis of the ring of polynomial invariants of $\mathfrak{g}$. In addition, all functions $f_{i, \lambda}$ commute with $H$.

A Poisson Isomorphism for $N$ invertible. We want to compare the Lie-Poisson bracket (3.1) on $\operatorname{Sym}(n, N)$ with that on $\mathfrak{s p}\left(n, N^{-1}\right)^{*}$.

To obtain the Lie-Poisson bracket on $\mathfrak{s p}\left(n, N^{-1}\right)^{*}$ we identify $\mathfrak{s p}\left(n, N^{-1}\right)^{*}$ with $\mathfrak{s p}\left(n, N^{-1}\right)$ via the invariant non-degenerate symmetric bilinear form

$$
\left\langle\left\langle Z_{1}, Z_{2}\right\rangle\right\rangle:=\operatorname{trace}\left(Z_{1} Z_{2}\right) .
$$

Therefore, the Lie-Poisson bracket on $\mathfrak{s p}\left(n, N^{-1}\right)^{*} \cong \mathfrak{s} \mathfrak{p}\left(n, N^{-1}\right)$ is given by

$$
\{\phi, \psi\}_{\mathfrak{s p}}(Z):=-\langle\langle Z,[\nabla \phi(Z), \nabla \psi(Z)]\rangle\rangle,
$$

where $\nabla$ is taken relative to $\langle\langle\cdot, \cdot\rangle\rangle$ and $\phi, \psi: \mathfrak{s p}\left(n, N^{-1}\right) \rightarrow \mathbb{R}$ are smooth functions.

In the following proposition, $\operatorname{Sym}(n, N)^{*}$ is identified with itself using the non-invariant inner product $\langle\langle\cdot, \cdot\rangle($ see $(2.12))$.

Proposition 4.2 The map $Z \in\left(\mathfrak{s p}\left(n, N^{-1}\right),\{\cdot, \cdot\}_{\mathfrak{s p}}\right) \mapsto Z N \in\left(\operatorname{Sym}(n, N),\{\cdot, \cdot\}_{N}\right)$ is an isomorphism of Lie-Poisson spaces.

Proof. By Proposition 2.3, the map $\Phi:\left(\operatorname{Sym}(n, N),[\cdot, \cdot]_{N}\right) \rightarrow\left(\mathfrak{s p}\left(n, N^{-1}\right),[\cdot, \cdot]\right)$ given by $\Phi(X):=N X$ is a Lie algebra isomorphism. Therefore its dual $\Phi^{*}:\left(\mathfrak{s p}\left(n, N^{-1}\right),\{\cdot, \cdot\}_{\mathfrak{s p}}\right) \rightarrow\left(\operatorname{Sym}(n, N),\{\cdot, \cdot\}_{N}\right)$ is an isomorphism of Lie-Poisson spaces (see, e.g., [16]). Since for any $Z \in \mathfrak{s p}\left(n, N^{-1}\right)$ and $Y \in \operatorname{Sym}(n, N)$ we have

$$
\left\langle\left\langle\Phi^{*}(Z), Y\right\rangle\right\rangle=\langle\langle Z, \Phi(Y)\rangle\rangle=\langle\langle Z, N Y\rangle=\operatorname{trace}(Z N Y)=\langle\langle Z N, Y\rangle\rangle
$$

it follows that $\Phi^{*}(Z)=Z N$. 
Since $N$ is invertible, as we have seen in $\S 3, \operatorname{Sym}(n, N)^{*}$ can be identified with itself using the ad-invariant inner product $\kappa_{N}$. To compute the pull-back $\Phi^{\dagger}: \mathfrak{s p}\left(n, N^{-1}\right) \rightarrow \operatorname{Sym}(n, N)$ if we identify $\operatorname{Sym}(n, N)^{*}$ with itself using $\kappa_{N}$, let $Z \in \mathfrak{s p}(n, N)$ and $Y \in \operatorname{Sym}(n, N)$. We get

$$
\kappa_{N}\left(\Phi^{\dagger}(Z), Y\right)=\langle\langle Z, \Phi(Y)\rangle\rangle=\left\langle\langle Z, N Y\rangle=\operatorname{trace}(Z N Y)=\kappa_{N}\left(N^{-1} Z, Y\right)\right.
$$

and hence

$$
\Phi^{\dagger}(Z)=N^{-1} Z
$$

The Mischenko-Fomenko System on $\left(\mathfrak{s p}\left(n, N^{-1}\right),\{\cdot, \cdot\}_{\mathfrak{s p}}\right)$. We now show that for $N$ with distinct eigenvalues $\Phi^{*}$ maps the system (1.1) to a Mischenko-Fomenko system on $\left(\mathfrak{s p}\left(n, N^{-1}\right),\{\cdot, \cdot\}_{\mathfrak{s p}}\right)$. Indeed, denoting $X:=\Phi^{*}(Z)=Z N$, we get

$$
\begin{aligned}
\dot{Z} & =\dot{X} N^{-1}=\left[X^{2}, N\right] N^{-1}=X^{2}-N X^{2} N^{-1} \\
& =Z N Z N-N Z N Z N N^{-1}=[Z, N Z N] .
\end{aligned}
$$

The following lemma, which can easily be verified, shows that the linear invertible operator $C: \mathfrak{s p}\left(n, N^{-1}\right) \rightarrow$ $\mathfrak{s p}\left(n, N^{-1}\right)$ defined by $C(Z)=N Z N$ is a sectional operator.

Lemma 4.3 The map $C$

(i) is well-defined, i.e. $N Z N$ indeed belongs to $\mathfrak{s p}\left(n, N^{-1}\right)$,

(ii) is symmetric relative to $\langle\langle\cdot, \cdot\rangle\rangle$,

(iii) satisfies $\left[C(Z), N^{-1}\right]=[N, Z]$,

(iv) is of the form $C_{a, b, D}$ with $a=N^{-1}, b=-N$, and $D$ having the same formula as $C$ on the Cartan algebra.

Applying the Mischenko-Fomenko Theorem 4.1 we get the following result.

Proposition 4.4 Let $N$ be invertible with distinct eigenvalues. The system

$$
\dot{Z}=[Z, N Z N]
$$

is integrable on the maximal dimensional orbits of $\mathfrak{s p}\left(n, N^{-1}\right)$ and its generically independent integrals in involution are the non-trivial coefficients of $\lambda^{i}$ in the polynomial expansion of $\frac{1}{k} \operatorname{tr}\left(Z+\lambda N^{-1}\right)^{k}$ that are not Casimir functions, $k=2, \ldots, n$. The Hamiltonian for $(4.6)$ is $H(Z):=\operatorname{trace}\left((Z N)^{2}\right) / 2$.

Pushing forward $Z$ by the map $\Phi^{*}$ we obtain the following statement.

Theorem 4.5 Let $N$ be invertible with distinct eigenvalues. The equation $\dot{X}=\left[X^{2}, N\right]$ is an integrable Hamiltonian system on the maximal dimensional symplectic leaf of $\operatorname{Sym}(n, N)$ defined by the function $l(X)=$ $\operatorname{tr}\left(X^{2}\right) / 2$ relative to the Lie-Poisson bracket (3.1). The independent integrals in involution are the nontrivial coefficients of $\lambda^{i}$ in the polynomial expansion of $\frac{1}{k} \operatorname{tr}\left(X N^{-1}+\lambda N^{-1}\right)^{k}$ that are not Casimir functions, $k=2, \ldots, n$.

The Mischenko-Fomenko System on the Dual of $\operatorname{Sym}(n)$. For $N$ invertible we can also show that our system (1.1) is a system of Mischenko-Fomenko type directly on $\operatorname{Sym}(n, N)$ viewed as its own dual under the ad-invariant inner product $\kappa_{N}(X, Y)=\operatorname{trace}(N X N Y)$ defined in equation (2.13).

Recall from Proposition 2.3 the Lie algebra isomorphism

$$
\Phi: X \in\left(\operatorname{Sym}(n, N),[,]_{N}\right) \longmapsto Z:=N X \in\left(\mathfrak{s p}\left(n, N^{-1}\right),[,]\right) .
$$

It is easy to see that the ad-invariant inner product $\kappa_{N}$ on $\operatorname{Sym}(n, N)$ is pushed forward by $\Phi$ to the nondegenerate ad-invariant form given by the trace of the product on $\mathfrak{s p}\left(n, N^{-1}\right)$. Therefore, the pull back 
$\Phi^{\dagger}: \mathfrak{s p}\left(n, N^{-1}\right) \rightarrow \operatorname{Sym}(n, N)$, where $\operatorname{Sym}(n, N)^{*}$ is identified with itself using $\kappa_{N}$, is an isomorphism of Lie-Poisson spaces. Hence $\Phi^{\dagger}(Z)=N^{-1} Z$ maps the Mischenko-Fomenko system (4.6) on $\mathfrak{s p}\left(n, N^{-1}\right)$ to a Mischenko-Fomenko system on $\operatorname{Sym}(n, N)$. A direct computation shows that $M:=N^{-1} Z$ satisfies (3.8).

In the ensuing sections we provide a direct proof of integrability on $\operatorname{Sym}(n, N)$ for $N$ with distinct eigenvalues but not necessarily invertible, that is, $N$ has at most one zero eigenvalue. In the invertible case, we provide a different sequence of integrals and, in addition, derive a second Hamiltonian structure for the Mischenko-Fomenko system on $\mathfrak{s p}\left(n, N^{-1}\right)$.

\section{Lax Pairs with Parameter}

To prove that system (1.1) is integrable for any $N$ having distinct eigenvalues, we will compute its flow invariants. Bear it in mind that, by virtue of the isospectral representation (1.2), we already know that the eigenvalues of $X$, or alternatively, the quantities trace $X^{k}$ for $k=1,2, \ldots, n-1$, are invariants.

One way to compute additional invariants is to rewrite the system as a Lax pair with a parameter. One can do this in a fashion similar to that for the generalized rigid body equations (see [15]).

Theorem 5.1 Let $\lambda$ be a real parameter. The system (1.2) is equivalent to the following Lax pair system

$$
\frac{d}{d t}(X+\lambda N)=\left[X+\lambda N, N X+X N+\lambda N^{2}\right] .
$$

Proof. The proof is a computation. The only nontrivial power of $\lambda$ to check is the first. In fact, the coefficient of $\lambda$ on the right hand side of equation (5.1) is

$$
\begin{aligned}
& {[N, N X+X N]+\left[X, N^{2}\right]} \\
& \quad=N^{2} X+N X N-N X N-X N^{2}+X N^{2}-N^{2} X=0,
\end{aligned}
$$

which proves (5.1).

Manakov [15] noticed that the generalized rigid body equations $\dot{M}=[M, \Omega]$ (see $\S 4$ ), can be written as a Lax equation with a parameter in the form

$$
\frac{d}{d t}\left(M+\lambda J^{2}\right)=\left[M+\lambda J^{2}, \Omega+\lambda J\right]
$$

Note the following contrast with our setting: in the Manakov case the system matrix $M$ is in $\mathfrak{s o}(n)$ and the parameter $J$ is a symmetric matrix while in our case $X$ is symmetric and the parameter $N \in \mathfrak{s o}(n)$.

For the generalized rigid body the nontrivial coefficients of $\lambda^{i}, 0<i<k$ in the traces of the powers of $M+\lambda J^{2}$ then yield the right number of independent integrals in involution to prove integrability of the flow on a generic adjoint orbit of $S O(n)$ (identified with the corresponding coadjoint orbit). The case $i=0$ needs to be eliminated, because these are Casimir functions.

Similarly, in our case, the nontrivial coefficients of $\lambda^{i}, 0 \leq i \leq k$, in

$$
h_{k}^{\lambda}(X):=\frac{1}{k} \operatorname{trace}(X+\lambda N)^{k}, \quad k=1,2, \ldots, n-1
$$

yield the conserved quantities. The coefficient of $\lambda^{r}, 0 \leq r \leq k$, in (5.3) is

$$
\operatorname{trace} \sum_{|i|=k-r} \sum_{|j|=r} X^{i_{1}} N^{j_{1}} X^{i_{2}} \cdots X^{i_{s}} N^{j_{s}}, \quad r=0, \ldots, k, \quad k=1, \ldots, n-1,
$$

where $i=\left(i_{1}, i_{2}, \ldots i_{s}\right), j=\left(j_{1}, j_{2}, \ldots j_{s}\right)$ are multi-indices, $i_{q}, j_{q}=0,1, \ldots, k$, and $|i|=\sum_{q=1}^{s} i_{q},|j|=$ $\sum_{q=1}^{s} j_{q}$. The coefficient of $\lambda^{k}$ is the constant $N^{k}$ so it should not be counted. Thus we have $r<k$. In addition, since the trace of a matrix equals the trace of its transpose, $X \in \operatorname{Sym}(n, N)$, and $N \in \mathfrak{s o}(n)$, it follows that

$$
\operatorname{trace} X^{i_{1}} N^{j_{1}} X^{i_{2}} \cdots X^{i_{s}} N^{j_{s}}=(-1)^{|j|} \operatorname{trace} N^{j_{s}} X^{j_{s}} \cdots X^{i_{2}} N^{j_{1}} X^{i_{1}} .
$$


Therefore, if $r$ is odd, then necessarily

$$
\operatorname{trace} \sum_{|i|=k-r} \sum_{|j|=r} X^{i_{1}} N^{j_{1}} X^{i_{2}} \cdots X^{i_{s}} N^{j_{s}}=0
$$

and only for even $r$ we get an invariant. Thus, we are left with the invariants

$$
h_{k, 2 r}(X):=\operatorname{trace} \sum_{|i|=k-2 r} \sum_{|j|=2 r} X^{i_{1}} N^{j_{1}} X^{i_{2}} \cdots X^{i_{s}} N^{j_{s}}
$$

for $k=1, \ldots, n-1, i_{q}=1, \ldots, k, j_{q}=0, \ldots, k-1, r=0, \ldots,\left[\frac{k-1}{2}\right]$, where $[\ell]$ denotes the integer part of $\ell \in \mathbb{R}$.

The integrals (5.4) are thus the coefficients of $\lambda^{2 r}, 0<2 r<k$, in the expansion of $\frac{1}{k} \operatorname{trace}(X+\lambda N)^{k}$. For example, if $k=1$ or $k=2$ then we have one integral, the coefficient of $\lambda^{0}$. If $k=3$ or $k=4$, only the coefficients of $\lambda^{2}$ and $\lambda^{0}$ yield non-trivial integrals. If $k=5$ or $k=6$ it is the coefficients of $\lambda^{4}, \lambda^{2}$, and $\lambda^{0}$ that give non-trivial integrals. In general, for the power $k$ we have $\left[\frac{k+1}{2}\right]$ integrals. Recall that $k=1, \ldots, n-1$. If $n-1=2 \ell$, we have hence

$$
\begin{aligned}
& 1+1+2+2+\cdots+\left[\frac{n-1+1}{2}\right]+\left[\frac{n-1+1}{2}\right]=1+1+2+2+\cdots+\ell+\ell \\
&=\ell(\ell+1)=\frac{n-1}{2}\left(\frac{n-1}{2}+1\right)=\frac{n-1}{2} \frac{n+1}{2}
\end{aligned}
$$

integrals. If $n-1=2 \ell+1$ then we have

$$
\begin{aligned}
1+1 & +2+2+\cdots+\left[\frac{n-2+1}{2}\right]+\left[\frac{n-2+1}{2}\right]+\left[\frac{n-1+1}{2}\right] \\
& =1+1+2+2+\cdots+\ell+\ell+(\ell+1) \\
& =\ell(\ell+1)+(\ell+1)=(\ell+1)^{2}=\left(\frac{n}{2}\right)^{2}
\end{aligned}
$$

integrals. However,

$$
\left[\frac{n}{2}\right]\left[\frac{n+1}{2}\right]=\left\{\begin{aligned}
\frac{n-1}{2} \frac{n+1}{2}, & \text { if } n \text { is odd } \\
\left(\frac{n}{2}\right)^{2}, & \text { if } n \text { is even }
\end{aligned}\right.
$$

Concluding, we have

$$
\left[\frac{n}{2}\right]\left[\frac{n+1}{2}\right]
$$

invariants which are the coefficients of $\lambda^{2 r}, 0<2 r<k$, in the expansion of $\frac{1}{k} \operatorname{trace}(X+\lambda N)^{k}$ for $k=$ $1, \ldots, n-1$.

We now address the issue of whether or not these integrals are the right candidates to prove complete integrability of the system $\dot{X}=\left[X^{2}, N\right]$.

- If $N$ is invertible, then $n=2 p$ and hence

$$
\begin{aligned}
{\left[\frac{n}{2}\right]\left[\frac{n+1}{2}\right] } & =\left[\frac{2 p}{2}\right]\left[\frac{2 p+1}{2}\right]=p^{2}=\frac{1}{2}\left(2 p^{2}+p-p\right) \\
& =\frac{1}{2}\left(\operatorname{dim} \mathfrak{s p}\left(2 p, N^{-1}\right)-\operatorname{rank} \mathfrak{s p}\left(2 p, N^{-1}\right)\right)
\end{aligned}
$$

which is half the dimension of the generic adjoint orbit in $\mathfrak{s p}\left(2 p, N^{-1}\right)$. Therefore, these conserved quantities are the right candidates to prove that this system is integrable on the generic coadjoint orbit of $\operatorname{Sym}(n, N)$. This will be proved in the next sections. 
- If $N$ is non-invertible (which is equivalent to $d \neq 0$ ), then $n=2 p+d$ and hence

$$
\begin{aligned}
{\left[\frac{n}{2}\right]\left[\frac{n+1}{2}\right] } & =\left[\frac{2 p+d}{2}\right]\left[\frac{2 p+d+1}{2}\right] \\
& =\left(p+\left[\frac{d}{2}\right]\right)\left(p+\left[\frac{d+1}{2}\right]\right) \\
& =p^{2}+p\left(\left[\frac{d}{2}\right]+\left[\frac{d+1}{2}\right]\right)+\left[\frac{d}{2}\right]\left[\frac{d+1}{2}\right] \\
& =p^{2}+p d+\left[\frac{d}{2}\right]\left[\frac{d+1}{2}\right] .
\end{aligned}
$$

The right number of integrals is $p(p+d)$ according to Proposition 3.2, so this calculation seems to indicate that there are additional integrals. The situation is not so simple since there are redundancies due to the degeneracy of $N$. Note, however, that if $d=1$, then we do get the right number of integrals. We shall return to the study of the degenerate case in $\S 7$.

Remark. Recall that in the special case when $N$ is invertible, we found the sequence of integrals given in Theorem 4.5. Note that these integrals have a different form from the family of integrals in (5.4). This does not necessarily mean that the two sets of functions are functionally independent.

\section{Involution}

In this section we prove involution of the integrals found in the previous section for arbitrary $N \in \mathfrak{s o}(n)$.

Bi-Hamiltonian Structure. We begin with the following observation.

Proposition 6.1 The system $\dot{X}=X^{2} N-N X^{2}$ is Hamiltonian with respect to the bracket $\{f, g\}_{N}$ defined in (3.1) using the Hamiltonian $h_{2}(X):=\frac{1}{2}$ trace $\left(X^{2}\right)$ and is also Hamiltonian with respect to the compatible bracket $\{f, g\}_{F N}$ defined in (3.2) using the Hamiltonian $h_{3}(X):=\frac{1}{3} \operatorname{trace}\left(X^{3}\right)$.

Proof. We have already implicitly checked the first statement using Euler-Poincaré theory, but here is a direct verification. We want to show that the condition $\dot{f}=\left\{f, h_{2}\right\}_{N}$ for any $f$ determines the equations $\dot{X}=X^{2} N-N X^{2}$. First note that $\frac{d}{d t} f(X)=\operatorname{trace}(\nabla f(X) \dot{X})$. Second, since $\nabla h_{2}(X)=X$, the right hand side $\left\{f, h_{2}\right\}_{N}$ becomes, by (3.1),

$$
\begin{aligned}
\left\{f, h_{2}\right\}_{N}(X) & =-\operatorname{trace}[X(\nabla f(X) N X-X N \nabla f(X))] \\
& =-\operatorname{trace}\left(\nabla f(X) N X^{2}-\nabla f(X) X^{2} N\right) .
\end{aligned}
$$

Thus, $\dot{X}=X^{2} N-N X^{2}$ as required.

To show that the same system is Hamiltonian relative to the frozen Poisson bracket, we proceed in a similar way. Noting that $\nabla h_{3}(X)=X^{2}$, we get from (3.2)

$$
\begin{aligned}
\left\{f, h_{3}\right\}_{F N}(X) & =-\operatorname{trace}\left(\nabla f N X^{2}-X^{2} N \nabla f\right) \\
& =-\operatorname{trace}\left(\nabla f N X^{2}-\nabla f X^{2} N\right),
\end{aligned}
$$

and hence $\dot{X}=X^{2} N-N X^{2}$, as before. 
Involution. Next we begin the proof that the $\left[\frac{n}{2}\right]\left[\frac{n+1}{2}\right]$ integrals given in (5.4), namely

$$
h_{k, 2 r}(X):=\operatorname{trace} \sum_{|i|=k-2 r} \sum_{|j|=2 r} X^{i_{1}} N^{j_{1}} X^{i_{2}} \cdots X^{i_{s}} N^{j_{s}} \text {, }
$$

where $k=1, \ldots, n-1, i_{q}=1, \ldots, k, j_{q}=0, \ldots, k-1, r=0, \ldots,\left[\frac{k-1}{2}\right]$, are in involution. It will be convenient below to write the expansion of $h_{k}^{\lambda}$ starting with the highest power of $\lambda$, that is,

$$
h_{k}^{\lambda}(X)=\frac{1}{k} \operatorname{trace}(X+\lambda N)^{k}=\sum_{r=0}^{k} \lambda^{k-r} h_{k, k-r}(X) .
$$

As explained before, not all of these coefficients should be counted: roughly half of them vanish and the last one, namely, $h_{k, k}$, is the constant $N^{k}$. Consistently with our notation for the Hamiltonians, we set $h_{k}=h_{k, 0}$.

Firstly we require the gradients of the functions $h_{k}^{\lambda}$.

Lemma 6.2 The gradients $\nabla h_{k}^{\lambda}$ are given by

$$
\nabla h_{k}^{\lambda}(X)=\frac{1}{2}(X+\lambda N)^{k-1}+\frac{1}{2}(X-\lambda N)^{k-1} .
$$

Proof. We have for any $Y \in \operatorname{Sym}(n, N)$,

$$
\begin{aligned}
\left\langle\left\langle\nabla h_{k}^{\lambda}(X), Y\right\rangle\right\rangle & =\mathbf{d} h_{k}^{\lambda}(X) \cdot Y=\operatorname{trace}\left((X+\lambda N)^{k-1} Y\right) \\
& =\frac{1}{2} \operatorname{trace}\left(\left((X+\lambda N)^{k-1}+(X-\lambda N)^{k-1}\right) Y\right) .
\end{aligned}
$$

Since $\langle\langle\rangle$,$\rangle is non-degenerate on \operatorname{Sym}(n, N)$, the result follows.

\section{Proposition 6.3}

$$
B_{X}\left(\nabla h_{k}^{\lambda}(X)\right)=C_{X}\left(\nabla h_{k+1}^{\lambda}(X)\right)
$$

Proof. Using (3.3) we get

$$
\begin{aligned}
B_{X}\left(\nabla h_{k}^{\lambda}(X)\right)=X \nabla h_{k}^{\lambda}(X) N-N \nabla h_{k}^{\lambda}(X) X \\
=\frac{1}{2}\left[X(X+\lambda N)^{k-1} N+X(X-\lambda N)^{k-1} N\right. \\
\left.\quad-N(X+\lambda N)^{k-1} X-N(X-\lambda N)^{k-1} X\right] \\
=\frac{1}{2}\left[(X+\lambda N)^{k} N-\lambda N(X+\lambda N)^{k-1} N+(X-\lambda N)^{k} N+\lambda N(X-\lambda N)^{k-1} N\right. \\
\left.\quad \quad-N(X+\lambda N)^{k}+\lambda N(X+\lambda N)^{k-1} N-N(X-\lambda N)^{k}-\lambda N(X-\lambda N)^{k-1} N\right] \\
=\frac{1}{2}\left[(X+\lambda N)^{k} N+(X-\lambda N)^{k} N-N(X+\lambda N)^{k}-N(X-\lambda N)^{k}\right] \\
=\nabla h_{k+1}^{\lambda}(X) N-N \nabla h_{k+1}^{\lambda}(X)=C_{X}\left(\nabla h_{k+1}^{\lambda}(X)\right)
\end{aligned}
$$

by (3.4), which proves the formula.

Proposition 6.4 The functions $h_{k, k-r}$ satisfy the recursion relation

$$
B_{X}\left(\nabla h_{k, k-r}(X)\right)=C_{X}\left(\nabla h_{k+1, k-r}(X)\right) .
$$


Proof. Substituting (6.1) into (6.3) we obtain

$$
\sum_{r=0}^{k} \lambda^{k-r} B_{X}\left(\nabla h_{k, k-r}(X)\right)=\sum_{r=0}^{k+1} \lambda^{k+1-r} C_{X}\left(\nabla h_{k+1, k+1-r}(X)\right) .
$$

Since $\nabla h_{k+1, k+1}(X)=N^{k+1}$, formula (3.4) implies that $C_{X}\left(\nabla h_{k+1, k+1}(X)\right)=0$. Thus on the right hand side the sum begins at $r=1$. Changing the summation index on the right hand side from $r$ to $r-1$ and identifying the coefficients of like powers of $\lambda$ yields (6.4).

Remark. It is worth making a few remarks about Propositions 6.3 and 6.4. Note that unlike the similar recursion for the rigid body Manakov integrals (see e.g. [27] and [22]), our polynomial recursion relation (6.3) does not have a premultiplier $\lambda$ on the right-hand side and the polynomials on the left and right hand sides appear to be of different order. This cannot be and indeed is not so. Indeed, the highest order coefficient on the right hand side vanishes by virtue of following result.

Corollary 6.5 The functions $h_{k, k-1}(X)$ are Casimirs for the frozen Poisson structure, i.e.

$$
C_{X}\left(\nabla h_{k, k-1}(X)\right)=0
$$

for all $k$.

Proof. By $(6.1), h_{k, k-1}(X)=\operatorname{trace}\left(N^{k-1} X\right)$, so its gradient equals $\nabla h_{k, k-1}(X)=N^{k-1}$. So (3.4) immediately gives (6.5).

The recursion relations (6.4) for $r=0$ also imply the following relation between the Hamiltonians that can also be easily checked by hand.

\section{Corollary 6.6}

$$
B_{X}\left(\nabla h_{k}(X)\right)=C_{X}\left(\nabla h_{k+1}(X)\right)
$$

Example: An interesting nontrivial example of the recursion relation to check is $B_{X}\left(d h_{3,2}(X)\right)=C_{X}\left(d h_{4,2}(X)\right)$, where $h_{3,2}(X)=\operatorname{trace}\left(N^{2} X\right)$ and $h_{4,2}(X)=\operatorname{trace}\left(N^{2} X^{2}\right)+\frac{1}{2} \operatorname{trace}(N X N X)$. This example illustrates how the recursion relation works despite the apparent inconsistency in order.

Involution follows immediately, using the recursion relations.

Proposition 6.7 The invariants $h_{k, k-r}$ are in involution with respect to both Poisson brackets $\{f, g\}_{N}$ and $\{f, g\}_{F N}$.

Proof. The definition of the Poisson tensors $B_{X}$ and $C_{X}$ and the recursion relation (6.4) give

$$
\begin{aligned}
\left\{h_{k, k-r}, h_{l, l-q}\right\}_{N} & =\left\langle\left\langle\nabla h_{k, k-r}(X), B_{X}\left(\nabla h_{l, l-q}(X)\right)\right\rangle\right\rangle \\
& =\left\langle\left\langle\nabla h_{k, k-r}(X), C_{X}\left(\nabla h_{l+1, l-q}(X)\right)\right\rangle\right\rangle \\
& =\left\{h_{k, k-r}, h_{l+1, l-q}\right\}_{F N}=-\left\{h_{l+1, l-q}, h_{k, k-r}\right\}_{F N} \\
& =-\left\langle\left\langle\nabla h_{l+1, l-q}(X), C_{X}\left(\nabla h_{k, k-r}(X)\right)\right\rangle\right\rangle \\
& =-\left\langle\left\langle\nabla h_{l+1, l-q}(X), B_{X}\left(\nabla h_{k-1, k-r}(X)\right)\right\rangle\right\rangle \\
& =-\left\{h_{l+1, l-q}, h_{k-1, k-r}\right\}_{N}=\left\{h_{k-1, k-r}, h_{l+1, l-q}\right\}_{N}
\end{aligned}
$$

for any $k, l=1, \ldots, n-1, r=1, \ldots, k$ and $q=0, \ldots, l-1$. Of course, in these relations we assume that $k-r$ and $l-q$ are even, for if at least one of them is odd, the identity above has zeros on both sides. Repeated application of this relation eventually leads to Hamiltonians $h_{k, k-r}$ where either $k-r$ is a power of $\lambda$ that does not exist for $k$, in which case the Hamiltonian is zero, or one is led to $h_{0,0}$ which is constant. This shows that $\left\{h_{k, k-r}, h_{l, l-q}\right\}_{N}=0$ for any pair of indices.

In a similar way one shows that $\left\{h_{k, k-r}, h_{l, l-q}\right\}_{F N}=0$. 
Bi-Hamiltonian Structure on $\mathfrak{s p}\left(n, N^{-1}\right)$. Using the bi-Hamiltonian property of system (1.1) and the Poisson isomorphism in Proposition 4.2 we get the following statement.

Theorem 6.8 The Lie-Poisson isomorphism $Z \in\left(\mathfrak{s p}\left(n, N^{-1}\right),\{\cdot, \cdot\}_{\mathfrak{s p}}\right) \mapsto Z N \in\left(\operatorname{Sym}(n, N),\{\cdot, \cdot\}_{N}\right)$ induces a bi-Hamiltonian structure for the Mischenko-Fomenko equations (4.6) on $\mathfrak{s p}\left(n, N^{-1}\right)$. The second Hamiltonian structure is

$$
\{f, g\}_{N^{-1}}(Z)=-\operatorname{trace}\left(N^{-1}[\nabla f(Z), \nabla g(Z)]\right)
$$

for any $f, g \in C^{\infty}\left(\mathfrak{s p}\left(n, N^{-1}\right)\right.$ and the Hamiltonian corresponding to this Poisson structure is $h(Z)=$ trace $\left((Z N)^{3}\right) / 3$.

\section{Independence}

To complete the proof of integrability we need to show that the integrals $h_{k, 2 r}$ are independent. We will demonstrate this first in the generic case when $N$ is invertible with distinct eigenvalues.

By (5.4), the gradients of the integrals $h_{k, 2 r}$ have the form

$$
\nabla h_{k, 2 r}(X):=\sum_{|i|=k-2 r-1} \sum_{|j|=2 r} X^{i_{1}} N^{j_{1}} X^{i_{2}} \cdots X^{i_{s}} N^{j_{s}}
$$

where $k=1, \ldots, n-1, i_{q}=1, \ldots, k, j_{q}=0, \ldots, k-1, r=0, \ldots,\left[\frac{k-1}{2}\right]$.

The Generic Case. We consider the case $N$ invertible with distinct eigenvalues. Therefore $d=0$ and $n=2 p$. In this case we show that the integrals $h_{k, 2 r}$ given in (5.4) are independent, and hence the system (1.1) is integrable.

Theorem 7.1 For $N$ invertible with distinct eigenvalues, the integrals $h_{k, 2 r}$ given by equation (5.4) are independent.

Proof. We are concerned with the linear independence (in a generic sense) of $(7.1)$ where $k=1, \ldots, n-1$, $i_{q}=1, \ldots, k, j_{q}=0, \ldots, k-1$ and $r=0, \ldots\left[\frac{1}{2}(k-1)\right]$. We recall that $N$ is invertible with distinct eigenvalues and, without loss of generality, assume that $X$ is diagonal,

$$
X=\operatorname{diag} \mu \text {. }
$$

This reduces the statement of the theorem to a problem about the independence of polynomials in single matrix variable.

Now, we aim to prove a stronger statement: the terms

$$
v_{i, j}=X^{i_{1}} N^{j_{1}} X^{i_{2}} \cdots X^{i_{s}} N^{j_{s}}
$$

are independent for all multi-indices $i$ and $j$ in the above range. Note however that each $v_{i, j}$ is a $q$-degree polynomial in $\mu_{1}, \mu_{2}, \ldots, \mu_{n}$, where $q=k-2 r-1 \in\{0, \ldots, n-2\}$. Let

$$
\mathcal{H}_{q}=\left\{v_{i, j}|| i|=q,| j \mid \text { even }\right\} .
$$

Clearly, in a generic sense, if linear dependence exists, it must exist within the set $\mathcal{H}_{q}$. In other words, if we can prove that there is no linear dependence within each $\mathcal{H}_{q}$, we are done. (Note that since $k \leq n-1$ in the expression (7.1) there is no dependence of powers of $X$ on lower powers through the characteristic polynomial.)

There is nothing to prove for $q=0$. For $q=1$ we have

$$
\mathcal{H}_{1}=\left\{X N^{j} \mid j \text { even }\right\} \cup\left\{N^{j} X \mid j \text { even }\right\} .
$$


Suppose that there exists linear dependence in $\mathcal{H}_{1}$. Then there necessarily exist $\rho_{0}, \rho_{2}, \ldots, \rho_{n-2}$ and $\kappa_{0}, \kappa_{2}, \ldots, \kappa_{n-2}$, not all zero, such that

$$
X\left(\sum \rho_{2 j} N^{2 j}\right)+\left(\sum \kappa_{2 j} N^{2 j}\right) X=0=X R(N)+K(N) X=0 .
$$

Therefore,

$$
\mu_{a}[R(N)]_{a, b}+[K(N)]_{a, b} \mu_{b}=0, \quad a, b=1, \ldots, n .
$$

Generically (i.e., for all $\mu$ except for a set of measure zero) this can hold only if $R(N), K(N)=0$. But $\operatorname{deg} R, \operatorname{deg} K \leq n-1$ and, since the eigenvalues of $N$ are distinct, the degree of the minimal polynomial of $N$ is $n$. Therefore $K, R \equiv 0$, a contradiction. Hence there is no linear dependence.

We continue to $q=2$. Now

$$
\mathcal{H}_{2}=\left\{X^{i_{1}} N^{j_{1}} X^{i_{2}} N^{j_{2}} X^{i_{3}}: i_{1}+i_{2}+i_{3}=2, j_{1}+j_{2} \text { even }\right\} .
$$

Assume that there exist $\rho_{i, j}$, not all zero, such that

$$
\sum_{i, j} \rho_{i, j} X^{i_{1}} N^{j_{1}} X^{i_{2}} N^{j_{2}} X^{i_{3}}=0 .
$$

Therefore

$$
\sum_{i, j} \rho_{i, j} \sum_{b} \mu_{a}^{i_{1}} \mu_{b}^{i_{2}} \mu_{c}^{i_{3}}\left(N^{j_{1}}\right)_{a, b}\left(N^{j_{2}}\right)_{b, c}=0, \quad a, c=1, \ldots, n .
$$

Note that we want the above to hold for all real $\mu_{k}$, but this is possible only if

$$
0=\sum_{i, j} \rho_{i, j} \sum_{b}\left(N^{j_{1}}\right)_{a, b}\left(N^{j_{2}}\right)_{b, c}=\sum_{i, j} \rho_{i, j}\left(N^{j_{1}+j_{2}}\right)_{a, c}, \quad a, c=1, \ldots, n,
$$

thus

$$
\sum_{i, j} \rho_{i, j} N^{j_{1}+j_{2}}=0
$$

We again obtain a polynomial in $N^{2}$ of degree $<n / 2$, which cannot be zero: a contradiction.

We can continue for higher $s$ in an identical manner.

Hence, since we have involution and independence, we have proved the following.

Theorem 7.2 For $N$ invertible with distinct eigenvalues the system (1.1) is completely integrable.

Corollary 7.3 For $N$ odd dimensional with distinct eigenvalues and nullity one, the system (1.1) is completely integrable.

Proof. In this case we have $d=1$ and $n=2 p+1$. All eigenvalues are distinct with one of them being zero. The above proof of independence still holds, the only change being that the characteristic (and minimal) polynomial of $N$ is of form $N w\left(N^{2}\right)$, where $w$ is a polynomial of degree $(n-1) / 2$.

Remark. Independently $\mathrm{Li}$ and Tomei [14] have shown the integrability of the same system in precisely the two cases discussed in this paper employing different techniques; they use the loop group approach suggested by the Lax equation with parameter (5.1) and give the solution in terms of factorization and the Riemann-Hilbert problem.

\section{Linearization of the Flow}

We have demonstrated integrability of the system (1.1) for appropriate $N$ by showing involution and independence of a sufficient number of integrals. The purpose of this section is to analyze the linearization of this system on the Jacobi variety of the curve

$$
\operatorname{det}(z I-\lambda N-X)=0
$$

using the theory discussed in [3] and [11], for example (see also [12], [13], [9], [1]). 
Linearization on the Jacobian for $N$ Invertible and Generic. Let us denote $X(\lambda):=X+\lambda N$ and $Y(\lambda):=N X+X N+\lambda N^{2}$. For $N$ invertible with distinct eigenvalues $(n:=2 p)$, choose an orthonormal basis of $\mathbb{R}^{2 p}$ in which $N$ is written as

$$
N=\left[\begin{array}{cc}
0 & V \\
-V & 0
\end{array}\right]
$$

where $V$ is a real diagonal matrix whose entries are $v_{1}, \ldots, v_{p}$.

Denote by $x_{k, l}$ the entries of the matrix $X$ and put it in the form

$$
X=\left[\begin{array}{cc}
U & C \\
C^{T} & R
\end{array}\right]
$$

where $U \in \operatorname{Sym}(p), R \in \operatorname{Sym}(p)$, and $C \in \mathcal{M}_{p \times p}$. Then the matrix $Y(\lambda)$ can be written as

$$
Y(\lambda)=\left[\begin{array}{cc}
-\lambda V^{2}+V C^{T}-C V & V R+U V \\
-V U-R V & -\lambda V^{2}+C^{T} V-V C
\end{array}\right] .
$$

The plane algebraic curve (called a spectral curve), associated to each $X(\lambda)$, namely,

$$
\Gamma_{X(\lambda)}:=\{(\lambda, z) \in \mathbb{C} \times \mathbb{C} \mid \operatorname{det}(z I-X(\lambda))=0\},
$$

is preserved by the flow of (5.1); the functions which are defined by the coefficients of the characteristic polynomial $Q(\lambda, z)$ of $X(\lambda)$ are constants of motion of (5.1). Similarly, for each $X(\lambda)$ the isospectral variety of matrices $A_{X(\lambda)}$ defined by

$$
A_{X(\lambda)}:=\left\{X^{\prime}(\lambda) \mid X(\lambda) \text { and } X^{\prime}(\lambda) \text { have the same characteristic polynomial }\right\}
$$

is preserved by the flow of (5.1). Notice that the spectral curve and the isospectral variety depend on the values of the constants of motion only (i.e., on the vector $\mathbf{c}=\left(q_{k l}\right)$, where $q_{k l}$ is the coefficient of $\lambda^{k} z^{l}$ in $Q(\lambda, z))$. Sometimes one writes $\Gamma_{\mathbf{c}}$ and $A_{\mathbf{c}}$ instead of $\Gamma_{X(\lambda)}$ and $A_{X(\lambda)}$. Notice that the spectral curve $\Gamma_{\mathbf{c}}$ is non-singular for generic values of $\mathbf{c}$. Let $\bar{\Gamma}_{\mathbf{c}}$ be the compactification in the projective plane $\mathbb{P}_{\mathbb{C}}^{2}$ of $\Gamma_{\mathbf{c}}$. For generic values of $\mathbf{c}$ the projective curve $\bar{\Gamma}_{\mathbf{c}}$ is also non-singular. Let us compute the points at infinity of the spectral curve. The equation of the affine spectral curve is:

$$
z^{2 p}+v_{1}^{2} v_{2}^{2} \ldots v_{p}^{2} \lambda^{2 p}+Q_{1}(\lambda, z)=0,
$$

where the polynomial $Q_{1}(\lambda, z)$ has degree strictly less than $2 p$. Put $\lambda=\nu / z_{0}$ and $z=\zeta / z_{0}$. Now, set $z_{0}=0$ in the equation

$$
z_{0}^{2 p} Q\left(\nu / z_{0}, \zeta / z_{0}\right)=0
$$

of the projective spectral curve $\bar{\Gamma}_{\mathbf{c}}$. We get the points at infinity

$$
\left\{P_{1}, \ldots, P_{2 p}\right\}:=\bar{\Gamma}_{\mathbf{c}} \backslash \Gamma_{\mathbf{c}},
$$

with $P_{k+1}=\left(1, \beta_{k+1}, 0\right), k=0,1, \ldots, 2 p-1$, where

$$
\beta_{k+1}:=v^{1 / p} \exp \left(i \frac{(2 k+1) \pi}{2 p}\right) \quad \text { and } \quad v:=\left|v_{1} v_{2} \cdots v_{p}\right| .
$$

At each of these points the meromorphic functions $\lambda$ and $z$ on $\bar{\Gamma}_{\mathbf{c}}$ have a pole of order 1 . Note also that the genus of the plane curve $\bar{\Gamma}_{\mathbf{c}}$ is $g:=(p-1)(2 p-1)$ (the genus of a non-singular plane curve is given by the well-known formula $g=(n-1)(n-2) / 2$, where $n$ is the degree of the homogeneous polynomial equation of the curve; see also [11]).

Take now a generic value of the vector $\mathbf{c}$ such that $\Gamma_{\mathbf{c}}$ is non-singular and note that for generic $(\lambda, z) \in \Gamma_{\mathbf{c}}$, the eigenspace of $X(\lambda)$ with eigenvalue $z$ is one-dimensional. If we denote by $\Delta_{k l}(z, X(\lambda))$ the cofactor of the matrix $z I_{2 p}-X(\lambda)$ corresponding to the $(k, l)$-th entry then, the unique eigenvector of $X(\lambda)$ with eigenvalue $z$, normalized by $\xi_{1}=1$, is

$$
\xi(z, X(\lambda)):=\left(\xi_{1}, \ldots, \xi_{2 p}\right)^{T},
$$


where

$$
\xi_{k}=\Delta_{1 k}(z, X(\lambda)) / \Delta_{11}(z, X(\lambda)) .
$$

By [3], p. 187, when $X(\lambda, t)$ flows according to (5.1), the corresponding eigenvector $\xi(t):=\xi(z, X(\lambda, t))$ satisfies the autonomous equation

$$
\dot{\xi}+Y \xi=\rho \xi
$$

where $Y:=Y(\lambda, X(\lambda, t))$ and $\rho$ is the scalar function

$$
\rho:=\rho(\lambda, z, X(\lambda, t))=\sum_{l=1}^{2 p} Y(\lambda, X(\lambda, t))_{1 l} \Delta_{1 l}(z, X(\lambda, t)) / \Delta_{11}(z, X(\lambda, t)) .
$$

The role of the eigenvector $\xi$ is to define the divisor map

$$
i_{\mathbf{c}}: A_{\mathbf{c}} \rightarrow \operatorname{Div}^{d}\left(\bar{\Gamma}_{\mathbf{c}}\right), \quad X(\lambda) \mapsto \mathcal{D}_{X(\lambda)},
$$

where $\mathcal{D}_{X(\lambda)}$ is the minimal effective divisor on $\Gamma_{\mathbf{c}}$ such that

$$
\left(\xi_{k}\right)_{\Gamma_{\mathbf{c}}} \geq-\mathcal{D}_{X(\lambda)}, k=1, \ldots, 2 p .
$$

Here, $d:=\operatorname{deg}\left(\mathcal{D}_{X(\lambda)}\right)$ is independent of $X(\lambda) \in A_{\mathbf{c}}$ (for generic c we can assume $A_{\mathbf{c}}$ connected) and so, $\mathcal{D}_{X(\lambda)}$ defines an effective divisor of degree $d$ in $\bar{\Gamma}_{\mathbf{c}}$.

Now choose and fix a divisor $\mathcal{D}_{0} \in \operatorname{Div}^{d}\left(\bar{\Gamma}_{\mathbf{c}}\right)$, a basis $\left(\omega_{1}, \ldots, \omega_{g}\right)$ of holomorphic differentials on $\bar{\Gamma}_{\mathbf{c}}$, and consider the vector $\bar{\omega}:=\left(\omega_{1}, \ldots, \omega_{g}\right)^{T}$. One defines the linearizing map by

$$
j_{\mathbf{c}}: A_{\mathbf{c}} \rightarrow \operatorname{Jac}\left(\bar{\Gamma}_{\mathbf{c}}\right), \quad X \mapsto \int_{\mathcal{D}_{0}}^{\mathcal{D}_{X}} \bar{\omega}
$$

where $\operatorname{Jac}\left(\bar{\Gamma}_{\mathbf{c}}\right)$ denotes the Jacobian of the curve $\bar{\Gamma}_{\mathbf{c}}$.

The role of the function $\rho$ is to linearize the isospectral flow of $(5.1)$ on $A_{\mathbf{c}}$, that is, to be able to write

$$
\int_{\mathcal{D}_{X(0)}}^{\mathcal{D}_{X(t)}} \bar{\omega}=t \sum_{k=1}^{2 p} \operatorname{Res}_{P_{k}}(\rho(\lambda, z, X(\lambda, 0)) \bar{\omega}), \quad \mathcal{D}_{X(0)}=\mathcal{D}_{0},
$$

if it is possible. The Linearization Criterion in [3] p.195 says that this happens if and only if for each $X \in A_{\mathbf{c}}$ there exists a meromorphic function $\Phi_{X}$ on $\bar{\Gamma}_{\mathbf{c}}$ with

$$
\left(\Phi_{X}\right)_{\bar{\Gamma}_{\mathbf{c}}} \geq-\sum_{k=1}^{2 p} P_{k}
$$

such that for all $P_{k}$,

$$
\text { (Laurent tail of } \left.d \rho(\lambda, z, X) / d t \text { at } P_{k}\right)=\left(\text { Laurent tail of } \Phi_{X} \text { at } P_{k}\right) \text {; }
$$

see also [11].

Now we shall apply the linearization criterion to our case. Firstly, we have:

$$
\Delta_{11}(z, X(\lambda))=z^{2 p-1}+v_{2}^{2} \ldots v_{p}^{2} z \lambda^{2 p-2}+Q_{11}(z, \lambda)
$$

where the polynomial $Q_{11}(z, \lambda)$ has degree strictly less than $2 p-1$. Then we compute

$$
\Delta_{12}(z, X(\lambda))=M_{12}(z, \lambda)+Q_{12}(z, \lambda),
$$

where the polynomial $Q_{12}(z, \lambda)$ has degree strictly less than $2 p-2$ and the homogeneous polynomial

$$
M_{12}(z, \lambda)=-x_{1,2} z^{2 p-2}+\ldots+x_{p+1, p+2} v_{1} v_{2} v_{3}^{2} \ldots v_{p}^{2} \lambda^{2 p-2}
$$


has degree $2 p-2$. Similarly, we get for $l=3, \ldots, 2 p, l \neq p+1$,

$$
\Delta_{1 l}(z, X(\lambda))=M_{1 l}(z, \lambda)+Q_{1 l}(z, \lambda),
$$

where the polynomial $Q_{1 l}(z, \lambda)$ has degree strictly less than $2 p-2$ and the homogeneous polynomial $M_{1 l}(z, \lambda)$ has degree $2 p-2$. For $l=p+1$, we get

$$
\Delta_{1, p+1}(z, X(\lambda))=M_{1, p+1}(z, \lambda)+Q_{1, p+1}(z, \lambda),
$$

where the polynomial $Q_{1, p+1}(z, \lambda)$ has degree strictly less than $2 p-1$ and the homogeneous polynomial $M_{1, p+1}(z, \lambda)$ has degree $2 p-1$.

Let $z_{k}$ be a local parameter around the point at infinity $P_{k}, k=1, \ldots, 2 p$. The Laurent tail of $z$ at $P_{k}$ is $\beta_{k} / z_{k}$ and the Laurent tail of $\lambda$ at $P_{k}$ is $1 / z_{k}$. By using the formulas above we conclude that the Laurent tail of

$$
\Delta_{1 l}(z, X(\lambda)) / \Delta_{11}(z, X(\lambda)), \quad l=2, \ldots, 2 p
$$

at $P_{k}$ is zero, since this meromorphic function is holomorphic at $P_{k}$. Moreover, this function has a zero at $P_{k}$ for each $k=1, \ldots, 2 p$, and $l \neq p+1$ (note that on the denominator the constant term $\beta_{k}^{2 p-1}+\beta_{k} v_{2}^{2} \ldots v_{p}^{2}$ is non-zero for generic c).

Now we compute the Laurent tail of $d \rho(\lambda, z, X) / d t$ at $P_{k}$. We emphasize that $\rho$ only depends on $t$ through $X(\lambda)$. Firstly, we see that the Laurent tail of

$$
\frac{d}{d t}\left(\Delta_{1 l}(z, X(\lambda)) / \Delta_{11}(z, X(\lambda))\right), \quad l=2, \ldots, 2 p
$$

at each $P_{k}$ is zero, because this meromorphic function is holomorphic at $P_{k}, k=1, \ldots, 2 p$. Since

$$
Y_{11}=-\lambda v_{1}^{2}, \quad Y_{1 l}=v_{1} x_{l, p+1}-v_{l} x_{1, p+l} \quad \text { for } \quad l=2, \ldots, p
$$

and

$$
Y_{1, p+l}=v_{l} x_{1, l}+v_{1} x_{p+1, p+l} \quad \text { for } \quad l=1, \ldots, p,
$$

we conclude that the Laurent tail of

$$
\begin{aligned}
\frac{d}{d t} \rho(\lambda, z, X)=\sum_{l=2}^{2 p} & \frac{d}{d t} Y(\lambda, X(\lambda, t))_{1 l} \frac{\Delta_{1 l}(z, X(\lambda, t))}{\Delta_{11}(z, X(\lambda, t))} \\
& +\sum_{l=2}^{2 p} Y(\lambda, X(\lambda, t))_{1 l} \frac{d}{d t} \frac{\Delta_{1 l}(z, X(\lambda, t))}{\Delta_{11}(z, X(\lambda, t))},
\end{aligned}
$$

at each $P_{k}$ is zero for all $k=1, \ldots, 2 p$. Thus, the linearization criterion applies with $\Phi_{X}=0$. We have proved the following.

Theorem 8.1 For $N$ invertible with distinct eigenvalues the map $j_{\mathbf{c}}$ linearizes the isospectral flow of the system (5.1) on the Jacobian $\operatorname{Jac}\left(\bar{\Gamma}_{\mathbf{c}}\right)$.

Linearization on the Prym Variety for $N$ Invertible and Generic. Since $(X+\lambda N)^{T}=X-\lambda N$, we have

$$
Q(-\lambda, z)=Q(\lambda, z)
$$

Thus there is an involution $\tau: \bar{\Gamma}_{\mathbf{c}} \rightarrow \bar{\Gamma}_{\mathbf{c}}$ of the spectral curve defined by

$$
\tau(\lambda, z)=(-\lambda, z) .
$$

In homogeneous coordinates $\lambda=\nu / z_{0}, z=\zeta / z_{0}$ this involution is given by

$$
\tau\left(\nu, \zeta, z_{0}\right)=\left(-\nu, \zeta, z_{0}\right) .
$$


Notice that the involution $\tau$ has no fixed points at infinity $\left(z_{0}=0\right.$ and $\nu=0$ would imply $\zeta=0$ from the homogeneous equation of the curve). Thus, the fixed points are obtained from the equation

$$
Q(0, z)=0
$$

which is the characteristic polynomial of the symmetric matrix $X$. Generically, we obtain $2 p$ distinct points $Z_{1}, \ldots, Z_{2 p}$ as its fixed (ramification) points, where $Z_{k}=\left(0, z_{k}, 1\right), k=1, \ldots, 2 p$, with $z_{k}$ the (real) eigenvalues of the symmetric matrix $X$. By the Riemann-Hurwitz formula, the quotient (smooth) curve $C_{1}:=\bar{\Gamma}_{\mathbf{c}} / \tau$ has genus $g_{1}:=(p-1)^{2}$.

Associated to the double covering

$$
\bar{\Gamma}_{\mathbf{c}} \rightarrow C_{1}
$$

is the Prym variety $\operatorname{Prym}\left(\bar{\Gamma}_{\mathbf{c}} / C_{1}\right)$, with the property that $\operatorname{Jac}\left(\bar{\Gamma}_{\mathbf{c}}\right)$ is isogenous to

$$
\operatorname{Jac}\left(C_{1}\right) \times \operatorname{Prym}\left(\bar{\Gamma}_{\mathbf{c}} / C_{1}\right) .
$$

It follows that

$$
\operatorname{dim}\left(\operatorname{Prym}\left(\bar{\Gamma}_{\mathbf{c}} / C_{1}\right)\right)=g-g_{1}=p^{2}-p .
$$

Let us denote by $\Omega_{\bar{\Gamma}_{\mathbf{c}}}$ the sheaf of holomorphic 1-forms on $\bar{\Gamma}_{\mathbf{c}}$. Recall that

$$
\operatorname{Jac}\left(\bar{\Gamma}_{\mathbf{c}}\right) \cong H^{0}\left(\bar{\Gamma}_{\mathbf{c}}, \Omega_{\bar{\Gamma}_{\mathbf{c}}}\right)^{*} / H_{1}\left(\bar{\Gamma}_{\mathbf{c}}, \mathbb{Z}\right) .
$$

The involution $\tau$ acts on the vector space $H^{0}\left(\bar{\Gamma}_{\mathbf{c}}, \Omega_{\bar{\Gamma}_{\mathbf{c}}}\right)$ and on the free group $H_{1}\left(\bar{\Gamma}_{\mathbf{c}}, \mathbb{Z}\right)$ having eigenvalues \pm 1 . The Prym variety $\operatorname{Prym}\left(\bar{\Gamma}_{\mathbf{c}} / C_{1}\right)$ can be equivalently described as the quotient

$$
H^{0}\left(\bar{\Gamma}_{\mathbf{c}}, \Omega_{\bar{\Gamma}_{\mathbf{c}}}\right)^{-*} / H_{1}\left(\bar{\Gamma}_{\mathbf{c}}, \mathbb{Z}\right)^{-}
$$

where the upper \pm index on a vector space denotes the \pm 1 eigenspaces.

Note that

$$
\begin{aligned}
\rho:=\rho(\lambda, z, X(\lambda, t))= & \sum_{l=1}^{2 p} Y(\lambda, X(\lambda, t))_{1 l} \Delta_{1 l}(z, X(\lambda, t)) / \Delta_{11}(z, X(\lambda, t)) \\
& =-\lambda v_{1}^{2}+\rho_{1}(\lambda, z, X(\lambda, t)),
\end{aligned}
$$

where the meromorphic function $\rho_{1}(\lambda, z, X(\lambda, t))$ has residue zero at each $P_{k}$; see the computation above. By [11], or by direct computation, we have

$$
\operatorname{Res}_{P_{k}}(\tau \rho(\lambda, z, X(\lambda, 0)))=-\operatorname{Res}_{P_{k}}(\rho(\lambda, z, X(\lambda, 0))) .
$$

It follows that the flow is actually linearized on $\operatorname{Prym}\left(\bar{\Gamma}_{\mathbf{c}} / C_{1}\right)$. Thus we have proved:

Corollary 8.2 For $N$ invertible with distinct eigenvalues the map $j_{\mathbf{c}}$ linearizes the isospectral flow of the system (5.1) on the Prym variety $\operatorname{Prym}\left(\bar{\Gamma}_{\mathbf{c}} / C_{1}\right)$.

The Case of $N$ Maximal Rank and Nullity One. Let us consider now the case of $n$ odd and $N$ having distinct eigenvalues and nullity one, i.e., $n=2 p+1$ and $\operatorname{rank} N=2 p$. Choose an orthonormal basis of $\mathbb{R}^{2 p+1}$ in which $N$ is written as

$$
N=\left[\begin{array}{ccc}
0 & V & 0 \\
-V & 0 & 0 \\
0 & 0 & 0
\end{array}\right],
$$

where $V$ is a real diagonal matrix whose entries are $v_{1}, \ldots, v_{p}$. The equation of the affine spectral curve is:

$$
z^{2 p+1}+v_{1}^{2} v_{2}^{2} \ldots v_{p}^{2} \lambda^{2 p} z+Q_{1}^{0}(\lambda, z)=0,
$$


where the polynomial $Q_{1}^{0}(\lambda, z)$ has degree strictly less than $2 p+1$. Put $\lambda=\nu / z_{0}$ and $z=\zeta / z_{0}$. Now set $z_{0}=0$ in the equation

$$
z_{0}^{2 p+1} Q\left(\nu / z_{0}, \zeta / z_{0}\right)=0
$$

of the projective spectral curve $\bar{\Gamma}_{\mathbf{c}}$. We get the points at infinity

$$
\left\{P_{0}, P_{1}, \ldots, P_{2 p}\right\}:=\bar{\Gamma}_{\mathbf{c}} \backslash \Gamma_{\mathbf{c}},
$$

with $P_{0}=(1,0,0)$ and $P_{k+1}=\left(1, \beta_{k+1}, 0\right), k=0,1, \ldots, 2 p-1$, where

$$
\beta_{k+1}:=v^{1 / p} \exp \left(i \frac{(2 k+1) \pi}{2 p}\right) \quad \text { and } \quad v:=\left|v_{1} v_{2} \cdots v_{p}\right| .
$$

Note that at each of these points, with the exception of $P_{0}$, the meromorphic functions $\lambda$ and $z$ on $\bar{\Gamma}_{\mathbf{c}}$ have a pole of order 1 . At $P_{0}$, the function $\lambda$ has a pole of order 1 and $z$ has a zero of order 1 . that

We shall analyze below in detail the particular case $p=2$ (that is, $n=5$ ). A direct computation shows

$$
\begin{aligned}
& \Delta_{11}=\left(z^{4}+v_{2}^{2} z^{2} \lambda^{2}\right)+Q_{11}^{0}(z, \lambda), \quad \operatorname{deg} Q_{11}^{0}<4 \\
& \Delta_{12}=\left(v_{1} v_{2} x_{34} z \lambda^{2}-x_{12} z^{3}\right)+Q_{12}^{0}(z, \lambda), \quad \operatorname{deg} Q_{12}^{0}<3, \\
& \Delta_{13}=\left(-v_{1} v_{2}^{2} z \lambda^{3}-v_{1} z^{3} \lambda\right)+Q_{13}^{0}(z, \lambda), \quad \operatorname{deg} Q_{13}^{0}<4, \\
& \Delta_{14}=\left(v_{2} x_{12} z^{2} \lambda+v_{1} x_{34} z^{2} \lambda-x_{14} z^{3}-v_{1} v_{2} x_{23} z \lambda^{2}\right)+Q_{14}^{0}(z, \lambda), \quad \operatorname{deg} Q_{14}^{0}<3, \\
& \Delta_{15}=\left(-v_{1} v_{2}^{2} x_{35} \lambda^{3}+v_{2}^{2} x_{15} z \lambda^{2}-v_{1} x_{35} z^{2} \lambda+x_{15} z^{3}\right)+Q_{15}^{0}(z, \lambda), \quad \operatorname{deg} Q_{15}^{0}<3 .
\end{aligned}
$$

Let $z_{k}$ be a local parameter around the point at infinity $P_{k}, k=1, \ldots, 4$. The Laurent tail of $z$ at $P_{k}$ is $\beta_{k} / z_{k}$ and the Laurent tail of $\lambda$ at $P_{k}$ is $1 / z_{k}$. By using the formulas above we conclude that the Laurent tail of

$$
\Delta_{1 l}(z, X(\lambda)) / \Delta_{11}(z, X(\lambda)), \quad l=2, \ldots, 5
$$

at $P_{k}$ is zero, since this meromorphic function is holomorphic at $P_{k}$.

For $P_{0}$ the computation changes. Let $u$ be a local parameter around the point $P_{0}$. The Laurent tail of $z$ at $P_{0}$ is zero $\left(z\right.$ has a simple zero at $\left.P_{0}\right)$ and the Laurent tail of $\lambda$ at $P_{0}$ is $1 / u$. We shall emphasize the leading term for the Laurent tail of

$$
\begin{aligned}
& \Delta_{11}=v_{2}^{2}\left(x_{33} x_{55}-x_{35}^{2}\right) / u^{2}+\ldots \\
& \Delta_{12}=v_{1} v_{2}\left(x_{35} x_{45}-x_{34} x_{55}\right) / u^{2}+\ldots \\
& \Delta_{13}=v_{1} v_{2}^{2} x_{55} / u^{3}+\ldots \\
& \Delta_{14}=v_{1} v_{2}\left(x_{23} x_{55}-x_{25} x_{35}\right) / u^{2}+\ldots \\
& \Delta_{15}=-v_{1} v_{2}^{2} x_{35} / u^{3}+\ldots
\end{aligned}
$$

and we get

$$
\begin{aligned}
& \Delta_{13} / \Delta_{11}=\left(\frac{v_{1} x_{55}}{x_{33} x_{55}-x_{35}^{2}}\right) \frac{1}{u}+\ldots, \\
& \Delta_{15} / \Delta_{11}=\left(\frac{-v_{1} x_{35}}{x_{33} x_{55}-x_{35}^{2}}\right) \frac{1}{u}+\ldots,
\end{aligned}
$$

the other two quotients $\Delta_{12} / \Delta_{11}$ and $\Delta_{14} / \Delta_{11}$ being holomorphic around $P_{0}$.

As in the case of $n$ even, we have

$$
\begin{aligned}
\rho(\lambda, z, X)= & -v_{1}^{2} \lambda+\left(v_{1} x_{23}-v_{2} x_{14}\right) \frac{\Delta_{12}}{\Delta_{11}} \\
& +v_{1}\left(x_{11}+x_{33}\right) \frac{\Delta_{13}}{\Delta_{11}}+\left(v_{2} x_{12}+v_{1} x_{34}\right) \frac{\Delta_{14}}{\Delta_{11}}+v_{1} x_{35} \frac{\Delta_{15}}{\Delta_{11}}
\end{aligned}
$$


and hence

$$
\operatorname{Res}_{P_{0}} \rho=v_{1}^{2}\left(-1+\frac{\left(x_{11}+x_{33}\right) x_{55}-x_{35}^{2}}{x_{33} x_{55}-x_{35}^{2}}\right) .
$$

¿From the system (1.2) we get

$$
x_{11}+x_{33}=C_{1} \quad \text { and } \quad x_{55}=C_{2},
$$

where $C_{1}, C_{2}$ are constants of the motion. Then a direct computation shows that

$$
\operatorname{Res}_{P_{0}} \frac{d \rho}{d t}=\frac{2 v_{1}^{2} C_{2} x_{35} \dot{x}_{35}\left(C_{1}-x_{33}\right)}{\left(C_{2} x_{33}-x_{35}^{2}\right)^{2}}
$$

which is non-zero generically. By applying Lemma 5.11 in [3] and the linearization criterion, we get the following result.

Proposition 8.3 For $N \in \mathfrak{s o}(5)$ having distinct eigenvalues and nullity one, generically the map $j_{\mathbf{c}}$ does not linearize the isospectral flow of the system (5.1) on the Jacobian $\operatorname{Jac}\left(\bar{\Gamma}_{\mathbf{c}}\right)$.

An easier computation gives the same result in the case $n=3$. We carried out the case $n=5$ as more representative of the general case; for $n=3$, there are various non-typical simplifications of the computations leading to the non-linearizability result due to the low size of the matrices involved.

We expect however that it will be possible to analyze linearization of the general case where $N$ has distinct eigenvalues (i.e. either $n=2 p$ and $N$ is invertible or, $n=2 p+1$, rank $N=2 p$ and $N$ has nullity one) on the generalized Jacobian (see e.g. [26]). To do this we intend to follow [10] and [4] (see also [2], [8] and [3]). We intend to carry out this study of generalized algebraic integrability of our system in a future publication.

Acknowledgments. We thank G. Prasad for his observation regarding Lie algebras. Luc Haine and Pol Vanhaecke have our gratitude for many very illuminating discussions regarding algebraic complete integrability. We also thank Alexey Bolsinov, Percy Deift, Igor Dolgachev, Michael Gekhtman, Rob Lazarsfeld, Alejandro Uribe, and Nguyen Tien Zung for useful conversations that clarified various points in the paper and thereby improved our exposition. Finally we would like to thank the referee for an extraordinarily useful and insightful referee report and for pointing out reference [27] and we would also like to thank the editor for his very helpful input.

\section{References}

[1] Adams, M.R., J. Harnad and J. Hurturbise [1993] Darboux coordinates and Liouville-Arnold integration in loop algebras, Comm. Math. Phys. 155, 385-413.

[2] Adler, M. and P. van Moerbeke [1980], Linearization of Hamiltonian systems, Jacobi varieties and representation theory, Advances in Math. 38, 318-379.

[3] Adler, M., P. van Moerbeke, and P. Vanhaecke [2004], Algebraic Integrability, Painlevé Geometry and Lie algebras, volume 47 of Ergebnisse der Mathematik und ihrer Grenzgebiete, Springer-Verlag 2004.

[4] Beauville, A. [1990], Jacobiennes des courbes spectrales et systèmes hamiltoniens completement integrables, Acta Math., 164, 211-235.

[5] Bloch, A.M. and A. Iserles [2006], On an isospectral Lie-Poisson system and its Lie algebra, Foundations of Computational Mathematics 6, 121-144.

[6] Bolsinov, A.V. [1992] Compatible Poisson brackets on Lie algebras and completeness of families of functions in involution, Math. USSR. Izvestiya, 38(1),69-90. 
[7] Bolsinov, A. V. and A. V. Borisov [2002], Compatible Poisson brackets on Lie algebras, Mat. Zametki, 72(1), 11-34.

[8] Deift, P., L.C. Li, and C. Tomei [1989], Matrix Factorizations and Integrable Systems, Comm. Pure Appl. Math. XLII, 443-521.

[9] Dubrovin, B.A., S.P. Novikov, and I.M. Krichever [1989], Integrable Systems, Encyclopaedia of Mathematical Sciences, 4, Springer-Verlag, Berlin.

[10] Gavrilov, L. [1999], Generalized Jacobians of spectral curves and completely integrable systems, Math. Z. 230, 487-508.

[11] Griffiths, P. [1985] Linearizing flows and a cohomological interpretation of Lax equations American J. of Mathematics 107, 1445-1483.

[12] Krichever, I.M. [1977], Methods of algebraic geometry in the theory of nonlinear equations, Russ. Math. Surv. 32, 185-213.

[13] Krichever, I.M. and S.P. Novikov [1980], Holomorphich bundles over algebraic curves and nonlinear equations, Russ. Math. Surv. 35, 53-79.

[14] Li, L.-C. and C. Tomei [2006], The complete integrability of a Lie-Poisson system proposed by Bloch and Iserles, Intern. Math. Res. Notes, 64949, 1-19.

[15] Manakov, S.V. [1976], Note on the integration of Euler's equations of the dynamics of an $n$-dimensional rigid body, Funct. Anal. and its Appl., 10, 328-329.

[16] Marsden, J.E. and T.S. Ratiu [1994], Introduction to Mechanics and Symmetry, volume 17 of Texts in Applied Mathematics; Second Edition, second printing, Springer-Verlag, 2003.

[17] Meshcheryakov, M.V. [1983], A characterisitic property of the inertial tensor of a multidimensional solid body, Russian Mathematical Surverys., 38(5), 210-202.

[18] Mikhailov, A. V. and V. V. Sokolov [2000], Integrable ODEs on associative algebras, Comm. Math. Phys., 211(1), 231-251.

[19] Mishchenko, A.S. and A.T. Fomenko [1976], On the integration of the Euler equations on semisimple Lie algebras, Sov. Math. Dokl., 17, 1591-1593.

[20] Mischenko, A.S. and A.T. Fomenko [1978], Euler equations on finite-dimensional Lie groups Izvestija AN SSSR, 42(2), 396-415.

[21] Mischenko, A.S. and A.T. Fomenko [1979], Integration of Euler equations on semisimple Lie algebras, (in Russian), Trudy Seminara po Vekt. i Tenz. Analizu, 19, Moscow MGU, 3-94.

[22] Morosi, C. and L. Pizzocchero [1996], On the Euler equation: bi-Hamiltonian structure and integrals in involution, Lett. Math. Phys. 37, 117-135.

[23] Mumford, D. [1984], Tata Lectures on Theta. II, volume 43 of Progr. Math., Birkhäuser, Boston.

[24] Odesskii, A. V. and V. V. Sokolov [2006], Integrable matrix equations related to pairs of compatible associative algebras, J. Phys. A, 39(40), 12447-12456. 
[25] Ratiu, T.S. [1980], Involution theorems. In Kaiser, G. and J. Marsden, editors, Geometric Methods in Mathematical Physics, volume 775 of Springer Lecture Notes, pages 219-257.

[26] Serre, J.P. [1959], Groupes Algebriques et Corps de Classes, Hermann, Paris.

[27] Trofimov, V.V. and A. Fomenko [1995] Algebra and geometry of integrable Hamiltonian differential equations. Russian, Moskva, Faktorial.

[28] Vanhaecke, P. [1998], Integrable systems and symmetric products of curves. Math. Z., 227(1), 93-127.

[29] Vanhaecke, P. [2001], Integrable Systems in The Realm of Algebraic Geometry, second edition, volume 1638 of Lecture Notes in Mathematics, Springer-Verlag. 